Prophage

The latent form of a temperate bacteriophage, in which its genome is integrated into the bacterial chromosome without disrupting the bacterial cell.
Michael Smith Laboratories, The University of British Columbia, 301-2185 East Mall, Vancouver, British Columbia, V6T 124, Canada. Correspondence to B.B.F. e-mail: bfinlay@interchange.ubc.ca doi:10.1038/nrmicro2265 Published online

7 December 2009 Corrected online

24 December 2012

\title{
Molecular mechanisms of Escherichia coli pathogenicity
}

\section{Matthew A. Croxen and B. Brett Finlay}

Abstract |Escherichia coli is a remarkable and diverse organism. This normally harmless commensal needs only to acquire a combination of mobile genetic elements to become a highly adapted pathogen capable of causing a range of diseases, from gastroenteritis to extraintestinal infections of the urinary tract, bloodstream and central nervous system. The worldwide burden of these diseases is staggering, with hundreds of millions of people affected annually. Eight E. coli pathovars have been well characterized, and each uses a large arsenal of virulence factors to subvert host cellular functions to potentiate its virulence. In this Review, we focus on the recent advances in our understanding of the different pathogenic mechanisms that are used by various E. coli pathovars and how they cause disease in humans.

Certain isolates of Escherichia coli have been implicated in a wide range of diseases that affect either animals or humans worldwide. To date, eight pathovars and their mechanisms of disease have been extensively studied. These pathovars can be broadly classified as either diarrhoeagenic E. coli or extraintestinal E. coli (ExPEC) ${ }^{1}$. Six pathovars - enteropathogenic E. coli (EPEC), enterohaemorrhagic E. coli (EHEC), enterotoxigenic E. coli (ETEC), enteroinvasive E. coli (EIEC; including Shigella), enteroaggregative E. coli (EAEC) and diffusely adherent E. coli (DAEC) - are diarrhoeagenic, and two pathovars - uropathogenic E. coli (UPEC) and neonatal meningitis E. coli (NMEC) - are the most common ExPEC isolates (FIG. 1). Other pathovars have been identified, but their mechanisms of pathogenesis are not as well defined (BOX 1).

The pathogenic $E$. coli isolates share many virulence strategies. Adhesion to host cells is a requirement for all pathovars except EIEC and is frequently achieved through long appendages called fimbriae or pili. Following attachment, E. coli must subvert host cell processes, often using secreted proteins. Hijacking and manipulating host cell signalling pathways can result in the coordinated invasion of host cells, evasion of host immune responses and efficient colonization, and ultimately leads to disease (reviewed in REF. 2). Each pathovar has its own characteristic mechanisms of attaching to and exploiting host cells (see Supplementary information S1 (table)), although they often target the same host machinery. For overviews of the mechanisms of pathogenicity of the diarrhoeagenic and ExPEC pathovars, see FICS 2-5.
Many of the virulence factors associated with E. colimediated disease have been known for several years. Recently, we have begun to elucidate the key interactions between host proteins and these virulence factors, providing an insight, at the molecular level, into how they contribute to disease. In this Review, we summarize recent advances in our understanding of these virulence factors and how they are used by E. coli to cause disease in humans.

\section{Evolution of diverse pathogens}

The loss and gain of mobile genetic elements has a pivotal role in shaping the genomes of pathogenic bacteria. Horizontal gene transfer (HGT) is an important mechanism that rapidly disseminates new traits to recipient organisms. Acquiring these new traits is crucial in promoting the fitness and survival of a pathogen while it coevolves with its host ${ }^{3}$. Large clusters of virulence genes, called pathogenicity islands (PAIs), can be found on plasmids or integrated into the chromosome in pathogenic bacteria, but they are not found in non-pathogenic bacteria. PAIs are usually flanked by mobile genetic elements - bacteriophages, insertion sequences or transposons and often insert near tRNA genes. It is not surprising that many of the virulence traits that are present in E. coli are carried on PAIs as well as on plasmids and prophages (see Supplementary information S2 (table)). Although most prophages are defective, some can still form infectious particles ${ }^{4}$. Traits that are acquired by HGT can allow the recipient bacterium to colonize a new niche, and selective pressures select for variants that can survive these 


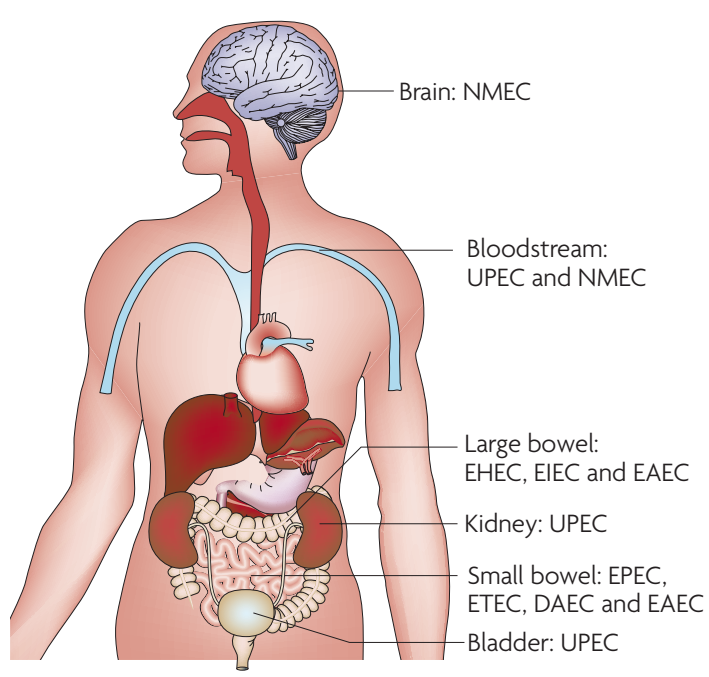

Figure 1| Sites of pathogenic Escherichia coli colonization. Pathogenic Escherichia coli colonize various sites in the human body. Enteropathogenic E. coli (EPEC), enterotoxigenic E. coli (ETEC) and diffusely adherent $E$. coli (DAEC) colonize the small bowel and cause diarrhoea, whereas enterohaemorrhagic E. coli (EHEC) and enteroinvasive E. coli (EIEC) cause disease in the large bowel; enteroaggegrative E. coli (EAEC) can colonize both the small and large bowels. Uropathogenic E. coli (UPEC) enters the urinary tract and travels to the bladder to cause cystitis and, if left untreated, can ascend further into the kidneys to cause pyelonephritis. Septicaemia can occur with both UPEC and neonatal meningitis E. coli (NMEC), and NMEC can cross the blood-brain barrier into the central nervous system, causing meningitis.

pressures. One theory is that multiple HGT events expose the bacteria to new selective pressures, which eventually select for more virulent organisms that become epidemic, such as EHEC and EIEC 5 . It should be noted that the evolution of pathovars might not always occur in a lineagespecific manner; for example, EHEC virulence factors were found to have been acquired independently by different phylogenies of E. coli ${ }^{6}$.

The genomes of the pathogenic E. coli are diverse and can be up to $1 \mathrm{Mb}$ larger than those of commensal isolates, mainly owing to the acquisition and loss of PAIs and other accessory genetic material. The sequenced $E$. coli isolates are thought to have a core genome of approximately 2,200 genes and a pan-genome of approximately 13,000 genes $^{7,8}$. It is intriguing that, although the genomes of most pathogenic $E$. coli isolates can encode more than 5,000 genes, less than half of these genes make up the core genome. This allows for substantial genetic diversity and plasticity in pathogenic isolates. For example, 13 genomic islands are found in the genome of UPEC str. CFT073, constituting almost $13 \%$ of the genome ${ }^{9}$. Interestingly, the distribution of virulence factors among other UPEC isolates is heterogeneous, and no one factor has been solely implicated in uropathogenesis. Comparative genomics has identified 131 UPEC-specific genes, most of which encode hypothetical proteins ${ }^{9}$. As these genes are specific to UPEC isolates, they might constitute a common subset that contributes to virulence.
The complete genome sequence of EPEC was recently published and found to encode approximately 400 more genes than $E$. coli $\mathrm{K}-12$ substrains, but around 650 fewer genes than EHEC O157:H7 and 770 fewer genes than UPEC str. CFT073 (REF. 10), suggesting that the repertoire of acquired virulence factors that are necessary to become EPEC might be substantially smaller than that required to create the other pathovars. Of the pathogenic E. coli, EIEC has endured the most recombination and has undergone patho-adaptation (reviewed in REF. 11), including the loss of genetic material. HGT of the virulence plasmid pINV gave EIEC the ability to be invasive; however, a deletion (such deletions are also referred to as black holes ${ }^{11}$ ) in a region of the genome that contains the lysine decarboxylase gene, $\underline{c a d A}$, was necessary for full fitness and adaptation to an intracellular lifestyle. Both gene loss and gain have contributed to the divergence and emergence of a diverse set of E. coli pathovars.

\section{Pathovars and pathogenesis}

Enteropathogenic Escherichia coli. EPEC is a major cause of potentially fatal diarrhoea in infants in developing countries ${ }^{1}$. This pathovar belongs to a family of pathogens that form attaching and effacing (A/E) lesions on intestinal epithelial cells; other members of the family include EHEC, rabbit diarrhoeagenic E. coli (RDEC), the murine pathogen Citrobacter rodentium and the recently identified Escherichia albertii (formerly known as Hafnia alvei), a pathogen that is associated with diarrhoea in humans. The attaching bacteria efface the microvilli and subvert host cell actin to form distinct pedestals beneath the site of attachment. This phenotype is afforded to EPEC by genes encoded on a $35 \mathrm{~kb}$ PAI known as the locus of enterocyte effacement (LEE) ${ }^{12}$. The LEE is highly regulated and encodes a type III secretion system (T3SS) that translocates bacterial effector proteins into the host cell cytoplasm. Seven effectors are encoded by the LEE, but there are several non-LEE encoded (Nle) effectors in addition to these ${ }^{13}$; the roles of many of these effectors are unknown.

The initial attachment of EPEC to enterocytes in the small bowel is thought to involve the bundle-forming pili that are encoded on the EPEC adherence factor (EAF) plasmid. Bundle-forming pili are rope-like fimbriae that interact both with other EPEC bacteria, to form microcolonies for localized adherence, and with $\mathrm{N}$-acetyl-lactosamine-containing receptors on host cell surfaces ${ }^{14}$. Recently, it was demonstrated that the E. coli common pilus, which is present in most E. coli isolates, may act in concert with bundle-forming pili to stabilize interactions between EPEC and host cells ${ }^{15}$. Intimate attachment is mediated through interactions of the bacterial outer-membrane protein intimin and the translocated intimin receptor (Tir) (FIG. 2). EPEC uses the T3SS to rapidly translocate Tir into the cytoplasm of host cells in a process that is possibly initiated through $\mathrm{Ca}^{2+}$ sensing ${ }^{16}$. Tir is then displayed on the surface of the host cell ${ }^{17}$ and acts as a receptor for intimin. Interactions with intimin lead to the clustering of Tir, which is then phosphorylated by various 


\section{Box 1 | Other human-pathogenic Escherichia coli}

In addition to the eight main Escherichia coli pathovars, other pathovars have been described but are not as well studied. Necrotoxigenic E. coli (NTEC) can be isolated from human extraintestinal infections such as urinary tract infections. NTEC secretes two cytotoxic necrotizing factors (CFN1 and CNF2), as well as cytolethal distending toxin. Cell-detaching E. coli (CDEC), which secretes CNF1 and a haemolysin, may be associated with diarrhoea in children ${ }^{1}$.

Perhaps the most interesting pathovar is adherent invasive $E$. coli (AIEC) as it has been implicated in $36 \%$ of ileal Crohn's disease. AIEC adheres to carcinoembryonic antigenrelated cell adhesion molecule 6 (CEACAM6) in the ileum ${ }^{135,136}$, which is overexpressed in patients who are predisposed to Crohn's disease. Adherence of AIEC also increases the expression of CEACAM6 through the stimulation of tumour necrosis factor and interferon- $\gamma$, which possibly promotes better colonization of the ileal mucosa and subsequent inflammation ${ }^{135}$. Following adhesion, AIEC can invade the intestinal epithelium. Individuals with Crohn's disease are often unable to control bacterial infections, possibly owing to defects in autophagy ${ }^{137}$, so AIEC can replicate quickly in epithelial cells and is inefficiently cleared by the innate immune response. AIEC can translocate through the intestinal epithelium into the lamina propria, where the bacteria interact and survive inside macrophages; they further exacerbate inflammation by stimulating tumour necrosis factor, eventually forming granulomas (reviewed in REF. 138).

\section{Filopodium \\ A thin, transient actin \\ protrusion that extends out from the cell surface and is formed by the elongation of bundled actin filaments.}

\section{Opsonophagocytosis} Increased uptake of bacteria by host cells owing to antibody or complement binding to the bacteria.

Haemolytic uraemic syndrome

A disease that primarily affects infants and children and is

characterized by the loss and destruction of red blood cells, sometimes leading to kidney failure.

\section{SOS response}

The bacterial response to DNA damage that is regulated by the LexA and recombinase A proteins and involves the expression of a network of $>40$ genes.

Macropinocytosis A form of regulated, actin-dependent endocytosis that involves the formation of large endocytic vesicles after the closure of cell surface membrane ruffles. host tyrosine kinases ${ }^{18-20}$. Experiments in vitro demonstrated that the phosphorylation of Tir recruits $\mathrm{Nck}^{21}$ to the site of attachment, which activates neural Wiskott-Aldrich syndrome protein (N-WASP) and the actin-related protein $2 / 3(\mathrm{ARP} 2 / 3$ ) complex to mediate actin rearrangements and pedestal formation $^{22}$. It was subsequently shown, however, that the phosphorylation of Tir is dispensable in A/E lesion formation ex vivo, as a Tir phosphorylation mutant can still recruit $\mathrm{N}$-WASP independently of $\mathrm{Nck}^{23}$. This exemplifies the importance of critically assessing the differences between phenotypes that are seen in vitro and what may actually occur in vivo.

EPEC has a large repertoire of effectors that are translocated into host cells by the T3SS and subvert host cell processes; for example, they cause cytoskeletal rearrangements and immune modulation, as well as contributing to diarrhoea (BOX 2; FIG. 2). Many of these translocated effectors have multiple functions. Mitochondrial-associated protein (Map) belongs to a family of proteins that share a WXXXE motif and was thought to mimic the active form of cell division control protein 42 (CDC42), a small G protein ${ }^{24}$. More recently, however, Map was shown to act as a guanine-nucleotide exchange factor for CDC42, regulating actin dynamics ${ }^{25}$ to result in the formation of the filopodia that surround bacterial microcolonies ${ }^{24}$. Map is also targeted to the mitochondria, where it disrupts mitochondrial structure and function ${ }^{26}$. A second multifunctional effector, EspF, is targeted to the mitochondria and triggers the mitochondrial death pathway ${ }^{27}$. In addition, EspF has been implicated in the inhibition of phagocytosis ${ }^{28}$ and the disruption of tight junction ${ }^{29}$ as well as in mimicking aspects of the host cell signalling pathway that is involved in membrane trafficking ${ }^{30}$. EspB (also known as EaeB) has a dual role as a T3SS translocation protein and as an effector that prevents phagocytosis ${ }^{31}$. Nle proteins also have roles in EPEC virulence. For example,
NleA (also known as EspI) reduces protein trafficking $^{32}$ and disrupts tight junctions ${ }^{33}$, EspI inhibits opsonophagocytosis by red blood cells ${ }^{34}$, and cycleinhibiting factor ( $\underline{\text { iff }}$ ) is a cyclomodulin that prevents progression of the cell cycle ${ }^{35}$ and, later, induces apoptosis ${ }^{36}$. Several other Nle proteins have been identified (reviewed in REF. 37); however, their characterization remains cursory.

Enterohaemorrhagic Escherichia coli. Cattle are a key reservoir for EHEC, which is a highly infectious $\mathrm{A} / \mathrm{E}$ pathogen that colonizes the distal ileum and large bowel in humans and is often the causative agent of outbreaks of severe gastroenteritis in developed countries. Transmission to humans usually occurs through contaminated food and water. In North America, Japan and parts of Europe, most outbreaks are due to EHEC serotype O157:H7, whereas other serotypes are important health concerns in other developed countries. Adults and children infected with EHEC suffer from haemorrhagic colitis (bloody diarrhoea), and further complications can lead to the potentially fatal haemolytic uraemic syndrome (HUS) ${ }^{1}$.

Almost all EHEC O157:H7 isolates harbour a $92 \mathrm{~kb}$ virulence plasmid called pO157, which has approximately 100 ORFs and encodes several virulence factors. However, the main virulence factor of EHEC is the phage-encoded Shiga toxin (Stx; also known as verocytotoxin), which is a defining characteristic of the Shiga toxin-producing E. coli (STEC) group to which EHEC O157:H7 belongs. There are two subgroups of Stx, Stx1 and Stx2, which can be found in various combinations in EHEC isolates, with Stx 2 being more prevalent in haemorrhagic colitis and HUS than Stx 1 (REF. 38). Stx is an $\mathrm{AB}_{5}$ toxin consisting of a pentamer of the $B$ subunit that is noncovalently bound to an enzymatically active A subunit. EHEC lacks a secretory mechanism for Stx, so the release of Stx occurs through lambdoid phage-mediated lysis in response to DNA damage and the SOS response ${ }^{39}$; antibiotic therapy should therefore be discouraged, as the toxin would be released.

The Stx receptors are the globotriaosylceramides (Gb3s) found on Paneth cells in the human intestinal mucosa ${ }^{40}$ (FIG. 2) and the surface of kidney epithelial cells $^{38}$. Cattle lack these receptors in the gastrointestinal tract, which may explain why EHEC colonization in cattle is asymptomatic ${ }^{41}$. The Stx B subunit interacts with Gb3 and induces membrane invaginations to facilitate internalization of the toxin ${ }^{42}$. The internalized Stx is trafficked through early endosomes into the Golgi, where the A subunit (an $\mathrm{N}$-glycosidase that prevents protein synthesis) is activated by a cleavage event ${ }^{43}$, leading to necrosis and cell death ${ }^{38}$. It should be noted that the physiological role of Stx binding to Paneth cells has not been explored. Interestingly, Stx can be found in Gb3-negative human intestinal cells (FIG. 2), possibly after being taken up by macropinocytosis ${ }^{44}$. Inside these cells, Stx does not prevent protein synthesis or induce apoptosis ${ }^{45}$ but, instead, is thought to dampen chemokine expression and therefore suppress inflammatory responses ${ }^{46}$. 


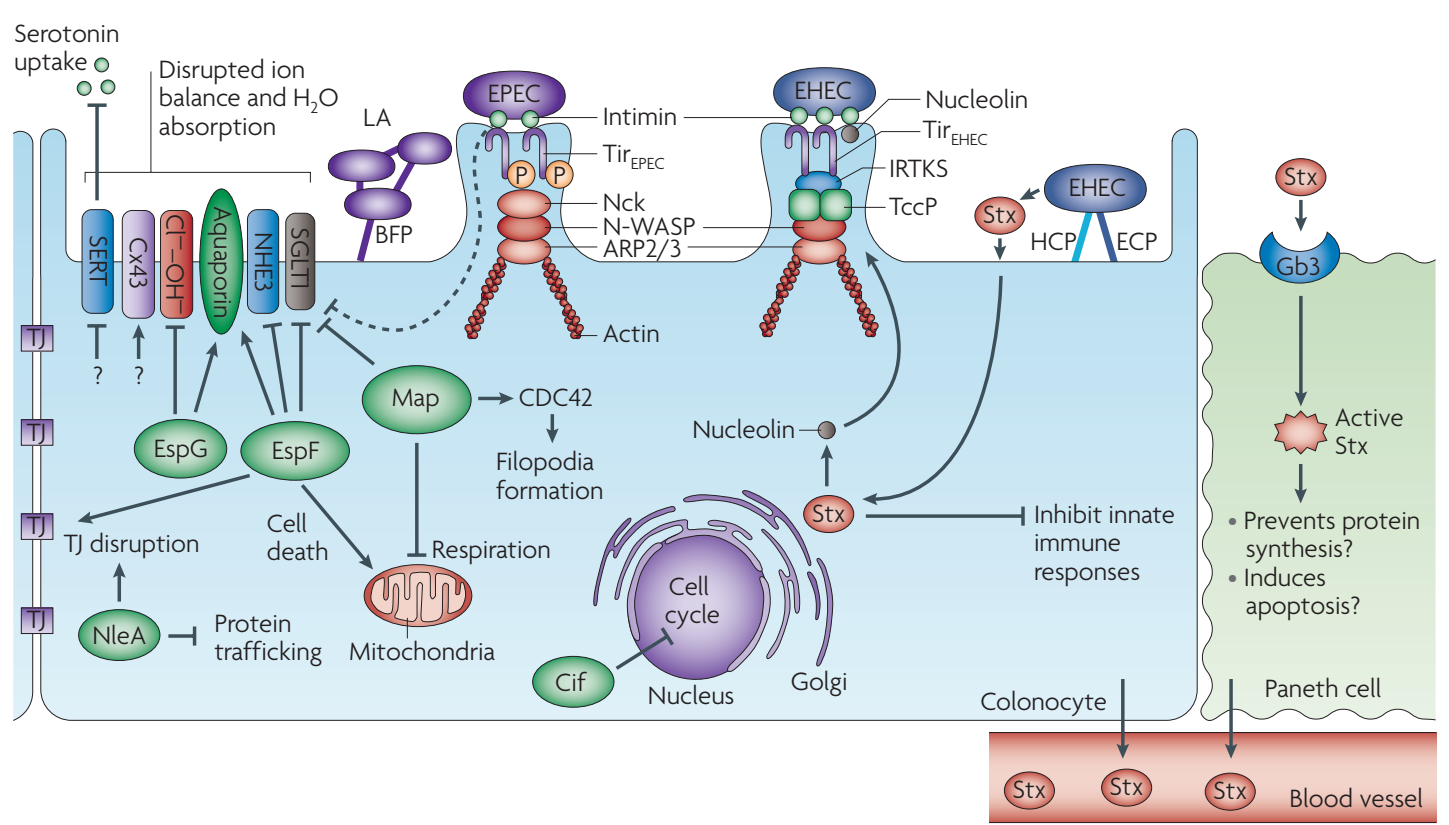

Figure 2 | Pathogenic mechanisms of enteropathogenic and enterohaemorrhagic Escherichia coli.

Enteropathogenic Escherichia coli (EPEC) and enterohaemorrhagic E. coli (EHEC) are attaching and effacing (A/E) pathogens that efface the microvilli and subvert host cell actin to form pedestals beneath the attachment site. The pedestal formation mechanisms shown for EPEC and EHEC are based on studies of the prototypical strains EPEC E2348/69 and EHEC O157:H7; lineage 2 EPEC strains and non-O157 EHEC strains can use a combination of these mechanisms for pedestal formation ${ }^{61}$. Effectors secreted by the type III secretion system can affect $\mathrm{Cl}^{-}-\mathrm{OH}^{-}$and $\mathrm{Na}^{+}-\mathrm{H}^{+}$ exchanger activity, mislocalize aquaporins and inhibit sodium-D-glucose cotransporter 1 (SCLT1). EPEC attaches to the small bowel through the bundle-forming pilus (BFP), forming localized adhesions (LA). Intimate attachment is mediated by the interaction between intimin and the translocated intimin receptor (Tir). Tir is phosphorylated by host tyrosine kinases, and phosphorylated Tir recruits Nck, which activates neural Wiskott-Aldrich syndrome protein (N-WASP) and the actinrelated protein 2/3 (ARP2/3) complex to mediate actin rearrangements and pedestal formation. Using the locus of the enterocyte effacement-encoded type III secretion system, a large repertoire of effector proteins is injected into the host cell, subverting host cell pathways. For full details, see main text and BOX 2. The mechanism of pedestal formation by EHEC is slightly different from that used by EPEC. Tir is not phosphorylated, and pedestal formation is Nck-independent. The actin rearrangements that are necessary for pedestal formation are mediated by Tir cytoskeleton-coupling protein (TccP; also known as $\mathrm{EspF}_{\mathrm{U}}$ ), which is linked to Tir through the host protein insulin receptor tyrosine kinase substrate (IRTKS; also known as BAIAP2L1) and interacts with N-WASP to activate the ARP2/3 complex. In addition to this intimate attachment, EHEC attaches to the large bowel through the E. coli common pilus (ECP) and the haemorrhagic coli pilus (HCP). EHEC injects many of the same effectors as EPEC into the host cell to manipulate host processes. In addition, Shiga toxin (Stx; also known as verocytotoxin) is released following phage-mediated lysis in response to stress, further contributing to disease. Globotriaosylceramides (Gb3s) on Paneth cells in the human intestinal mucosa act as receptors for Stx. For full details, see main text and BOX 2. CDC42, cell division control protein 42; Cx43, connexin 43 (also known as GJA1); Cif, cycle-inhibiting factor; Map, mitochondrial-associated protein; $\mathrm{NHE} 3, \mathrm{Na}^{+}-\mathrm{H}^{+}$exchanger 3; NleA, non-LEE-encoded effector A (also known as Espl); SERT, serotonin transporter; TJ, tight junctions.

The initial attachment of EHEC to colonocytes is not well defined. EHEC possesses 16 potential fimbrialike operons ${ }^{47}$; however, these have not been extensively studied. Recent work has identified a type IV pilus, called the haemorrhagic coli pilus ${ }^{48}$, that is involved in adherence and biofilm formation; flagella and the E. coli common pilus might also be involved in attachment to host cells ${ }^{49,50}$. As with EPEC, intimate attachment of EHEC to host cells occurs through interactions between intimin and Tir (FIG. 2). Attachment can also be enhanced by the interaction of intimin with nucleolin, a surface-localized intimin receptor, the expression of which is increased by Stx2 (REF. 51). As Stx is released on bacterial lysis, the increase in nucleolin expression may be important for the attachment of progeny EHEC.
The EHEC genome contains the same LEE as the EPEC genome, but EHEC injects around twice as many effectors into host cells as EPEC, most of which are redundant ${ }^{52}$. This redundancy may provide EHEC with an evolutionary advantage that allows it to outcompete other bacteria. The mechanism of pedestal formation by EHEC is slightly different from that of EPEC - Tir is not tyrosine phosphorylated by the host cell ${ }^{53}$, and pedestal formation is Nck-independent - although the pedestals themselves are highly similar. Subversion of the host cell actin cytoskeleton is mediated by an EspF homologue called Tir cytoskeleton-coupling protein (TccP; also known as $\left.\mathrm{EspF}_{\mathrm{U}}\right)^{54,55}$, which is linked to Tir by a host protein, insulin receptor tyrosine kinase substrate (IRTKS; also known as BAIAP2L1), a homologue of insulin 


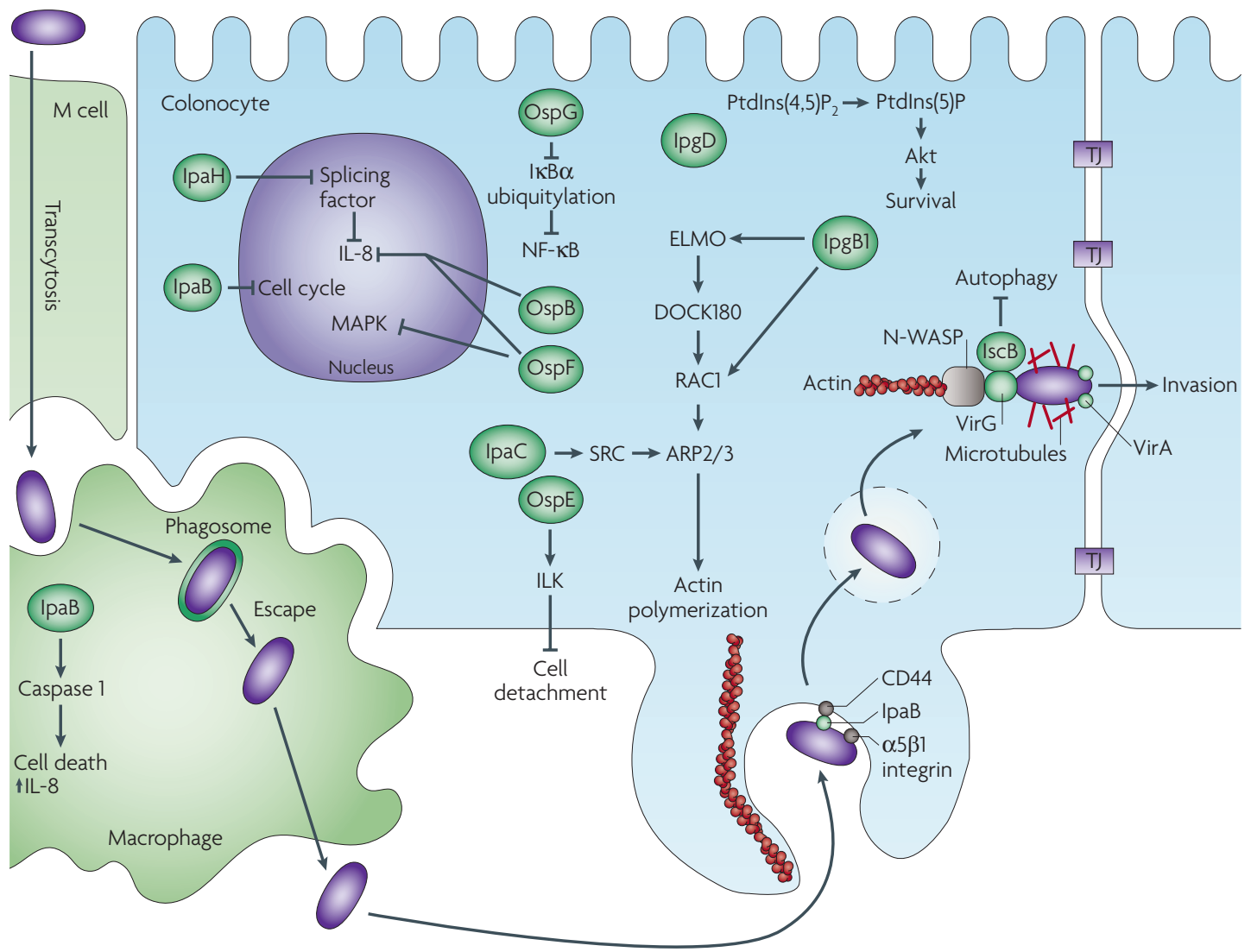

Figure 3 | Pathogenic mechanisms of Shigella (enteroinvasive Escherichia coli). Shigella gain access to the submucosa through microfold (M) cells and, following replication in macrophages, invade the basolateral side of colonocytes; all of these processes are achieved by effectors that are secreted into host cells by the type III secretion system. Once in the colonocyte cytoplasm, more effectors are injected to hijack host machinery, prevent detection by the host immune system and promote cell-to-cell dissemination of the bacterium. For full details, see main text. ARP2/3, actin-related protein 2/3; DOCK180, dedicator of cytokinesis protein 1; ELMO, engulfment and cell motility; I $\mathrm{kBa}$, inhibitor of NF-kB subunit- $\alpha$; IL-8, interleukin-8; ILK, integrin-linked kinase; MAPK, mitogen-activated protein kinase;

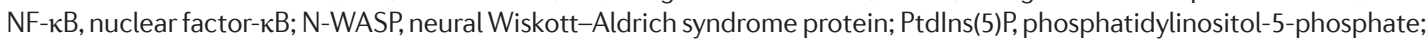
Ptdlns(4,5) $\mathrm{P}_{2}$, phosphatidylinositol-4,5-bisphosphate; TJ, tight junctions.

receptor substrate protein of $53 \mathrm{kDa}$ (IRSp53; also known as BAIAP2) $)^{56,57}$. TccP interacts with N-WASP to potently activate ARP2/3 complex-mediated actin assembly ${ }^{58,59}$; further details of these interactions have recently been reviewed ${ }^{60}$. It is important to note that the mechanisms described above for pedestal formation in EPEC and EHEC are representative of the prototypical strains; lineage 2 EPEC strains and non-O157 EHEC strains use a combination of the Nck-dependent and Nck-independent mechanisms (reviewed in REF. 61).

Intriguingly, EHEC can sense the hormones adrenaline and noradrenaline from host cells, as well as the quorum-sensing molecule auto-inducer 3 (AI-3) from gastrointestinal cells, to regulate motility and T3SS expression (reviewed in REF. 62). Sensing of these molecules is required for virulence of EHEC in animal models and presents a new interaction that should be taken into account when considering pathogen-host interactions.
Enterotoxic Escherichia coli. ETEC is the most common cause of travellers' diarrhoea and can have fatal consequences for children under 5 years of age. ETEC is also important in the farming industry, as post-weaning piglets are highly susceptible to infection ${ }^{38}$.

ETEC engagement with epithelial cells of the small bowel (FIG. 4) is mediated through colonization factors (CFs), which can be non-fimbrial, fimbrial, helical or fibrillar. A large number of CFs have been identified, of which CFA/I, CFA/II and CFA/IV are the most common ${ }^{63}$. The cognate receptors for CFs are poorly defined, although recent work has found interactions between CFA/I and carbohydrate moieties of non-acid glycosphingolipids and glycoproteins ${ }^{64}$ and between CFA/IV and the acid glycosphingolipid sulphatide ${ }^{65}$. A recent study demonstrated that flagella that are transiently bound at the tip with the secreted adhesin EtpA can be used as epithelial-cell adherence factors ${ }^{66}$. Both CFs and flagella anchor ETEC for initial attachment to host cells, but more intimate attachment may be facilitated by the outer-membrane proteins Tia and TibA ${ }^{63}$ (FIG. 4a). 
a ETEC

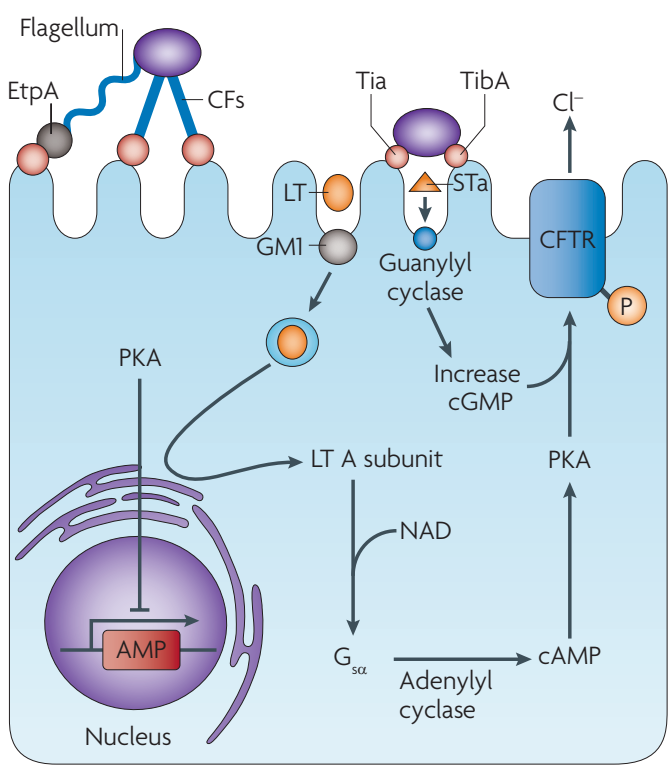

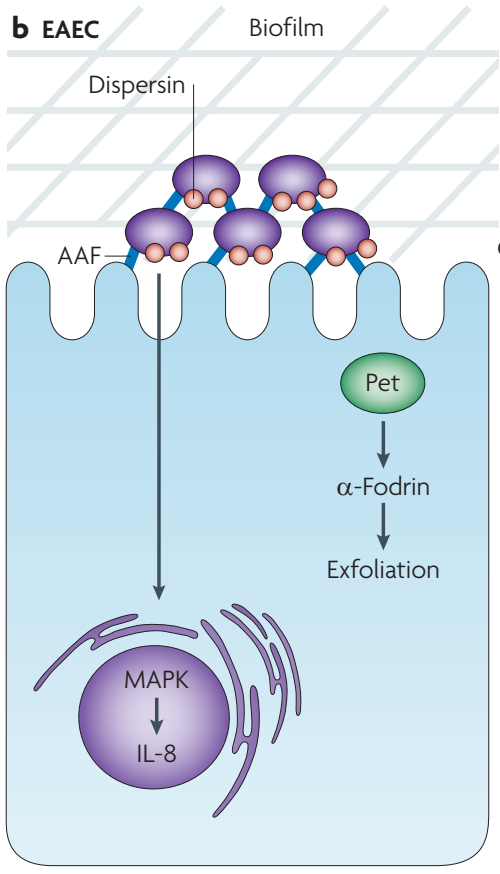

c DAEC

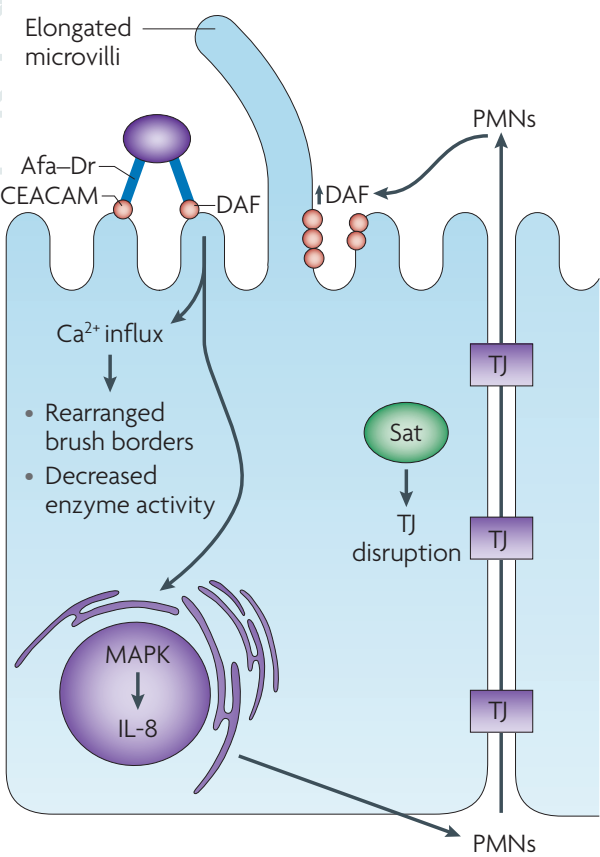

Figure 4 | Pathogenic mechanisms of enterotoxigenic, enteroagreggative and diffusely adherent Escherichia coli. a| Enterotoxigenic Escherichia coli (ETEC) becomes anchored to enterocytes of the small bowel through colonization factors (CFs) and an adhesin that is found at the tip of the flagella (EtpA). Tighter adherence is mediated through Tia and TibA. Two toxins, heat-labile enterotoxin (LT) and heat-stable enterotoxin (ST), are secreted and cause diarrhoea through cyclic AMP (cAMP)- and cyclic GMP (cGMP)-mediated activation of cystic fibrosis transmembrane conductance regulator (CFTR). $\mathbf{b}$ | Enteroagreggative E. coli (EAEC) attaches to enterocytes in both the small and large bowels through aggregative adherence fimbriae (AAF) that stimulate a strong interleukin-8 (IL-8) response, allowing biofilms to form on the surface of cells. Plasmid-encoded toxin (Pet) is a serine protease autotransporter of the Enterobacteriaceae (SPATE) that targets $\alpha$-fodrin (also known as SPTAN1), which disrupts the actin cytoskeleton and induces exfoliation. c|Diffusely adherent $E$. coli (DAEC) forms a diffuse attaching pattern on enterocytes of the small bowel, which is mediated through afimbrial (Afa) and fimbrial adhesins, which are collectively known as Afa-Dr fimbriae. Most Afa-Dr fimbriae bind to complement decay-accelerating factor (DAF); a subset of Afa-Dr fimbriae bind to receptors in the carcinoembryonicantigen-related cell-adhesion molecule (CEACAM) family. The autotransported toxin Sat has been implicated in lesions of tight junctions (TJs) in Afa-Dr-expressing DAEC, as well as in increased permeability. Polymorphonuclear leukocyte (PMN) infiltration increases surface localization of DAF. For full details, see main text. AMP, antimicrobial peptides; $\mathrm{G}_{\text {sa, }}$ stimulatory guanylyl-nucleotide-binding (G) protein $\alpha$-subunit; MAPK, mitogen-activated protein kinase; PKA, protein kinase A.

ETEC-mediated diarrhoea has been attributed to the secretion of heat-stable enterotoxins (STs), the heat-labile enterotoxin (LT) or a combination of these. STs are small toxins that can be further classed as STa or STb on the basis of structure and function ${ }^{1}$ and are synthesized as 72-amino-acid precursors that are processed into active forms of 18-19 amino acids for STa and 48 amino acids for STb. STa, which is associated with human disease, binds to guanylyl cyclase receptors on the brush border of the intestine and stimulates their activity. This leads to increased intracellular levels of cyclic GMP, resulting in impaired $\mathrm{Na}^{+}$absorption, as well as activation of the cystic fibrosis transmembrane conductance regulator (CFTR) ${ }^{63}$. LT is similar to the cholera toxin and is also an $\mathrm{AB}_{5}$ toxin. It is secreted from the pole of the bacterial cell ${ }^{67}$ and associates with lipopolysaccharide on the surface, where it may act as an adhesin, facilitating attachment to host cells ${ }^{68}$. The B subunit of LT interacts with the monosialoganglioside GM1 on host cells; the toxin is internalized at lipid rafts ${ }^{69}$, where it is trafficked to the cytosol through the endoplasmic reticulum. The A subunit ADP-ribosylates the stimulatory guanine-nucleotide-binding $(G)$ protein a-subunit $\left(\mathrm{G}_{\mathrm{sa}}\right)$, which activates adenylyl cyclase and increases the levels of intracellular cyclic AMP. This activates cAMP-dependent protein kinase A (PKA), which in turn activates $\mathrm{CFTR}^{38}$. Intriguingly, activation of PKA and other host cell signalling pathways by LT has also been shown to inhibit expression of antimicrobial peptides $^{70}$.

Other virulence factors have been shown to be secreted by ETEC (reviewed in REF. 63). For example, EatA is a serine protease autotransporter of the Enterobacteriaceae (SPATE) that cleaves cathepsin G and may accelerate fluid build-up. Other secreted virulence factors include CylA, a pore-forming cytotoxin, and E. coli ST1 (EAST1), which may have similar functions to STa. 

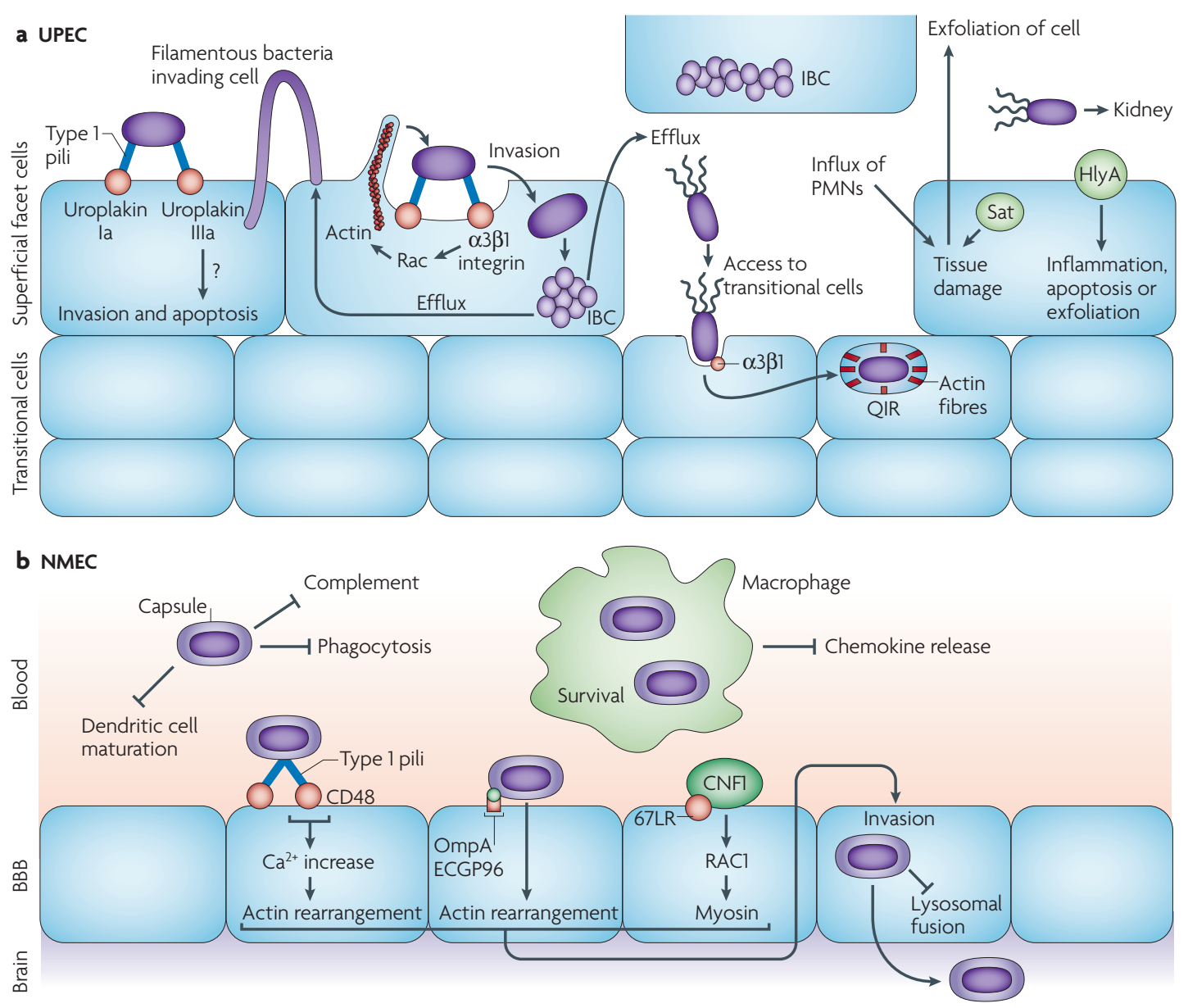

Figure 5 | Pathogenic mechanisms of extraintestinal Escherichia coli. The different stages of extraintestinal Escherichia coli infections are shown. a Uropathogenic E. coli (UPEC) attaches to the uroepithelium through type 1 pili, which bind the receptors uroplakin la and IIla; this binding stimulates unknown signalling pathways (indicated by the question mark) that mediate invasion and apoptosis. Binding of type 1 pili to $\alpha 3 \beta 1$ integrins also mediates internalization of the bacteria into superficial facet cells to form intracellular bacterial communities (IBCs) or pods. Sublytic concentrations of the pore-forming haemolysin $\mathrm{A}(\mathrm{HlyA})$ toxin can inhibit the activation of Akt proteins and leads to host cell apoptosis and exfoliation. Exfoliation of the uroepithelium exposes the underlying transition cells for further UPEC invasion, and the bacteria can reside in these cells as quiescent intracellular reservoirs (QIRs) that may be involved in recurrent infections. b| Neonatal meningitis E. coli (NMEC) is protected from the host immune response by its $\mathrm{K} 1$ capsule and outer-membrane protein A (OmpA). Invasion into macrophages may provide a replicative niche for high bacteraemia, allowing the generation of sufficient bacteria to cross the blood-brain barrier (BBB) into the central nervous system. Attachment of NMEC is mediated by type 1 pili binding to CD48 and OmpA binding to ECGP96. Invasion involves cytotoxic necrotizing factor 1 (CNF1) binding to $67 \mathrm{kDa}$ laminin receptor (67LR; also known as RPSA, as well as type1 pili and OmpA binding their receptors. For full details, see main text. PMN, polymorphonuclear leukocyte; Sat, secreted autotransporter toxin.

\section{Microfold cell}

A specialized epithelial cell that delivers antigens by transepithelial vesicular transport from the gut lumen directly to intraepithelial lymphocytes and subepithelial lymphoid tissues.

Inflammasome A large multiprotein complex that stimulates caspase-1dependent release of proinflammatory cytokines.
Enteroinvasive Escherichia coli. It is generally accepted that EIEC and Shigella should form a single pathovar, because they have the same mechanisms of pathogenicity. However, the genus name Shigella is still used owing to its association with the disease shigellosis and is retained in this section.

Shigella are highly infectious bacteria that cause bacillary dysentery and bloody diarrhoea ${ }^{1}$. This pathovar differs from the other E. coli pathovars, because it includes obligate intracellular bacteria that have neither flagella nor adherence factors. Virulence is largely due to a $220 \mathrm{~kb}$ plasmid that encodes a T3SS on the Mxi-Spa locus that is required for invasion, cell survival and apoptosis of macrophages (reviewed in REFS 71,72).
Infection commences in the colon, where the bacteria pass through microfold cells ( $\mathrm{M}$ cells) by transcytosis to reach the underlying submucosa (FIG. 3). The disruption of tight junctions and the damage that is caused by inflammation also give Shigella access to the submucosa. Shigella uptake into resident macrophages, escape from the phagosome, caspase-1-dependent inflammasome activation and ultimate release from macrophages have been extensively reviewed in REF. 72. Shigella are released from dead macrophages into the submucosa, from where they invade the basolateral side of colonocytes with the aid of effectors that are secreted by the T3SS. Key effectors, such as IpaC, activate SRC kinases at the site of bacterial contact to ultimately recruit the ARP $2 / 3$ complex 


\section{Box 2 | Mechanisms of A/E pathogen-induced diarrhoea}

The mechanisms of diarrhoea caused by attaching and effacing (A/E) pathogens (FIG. 2) have puzzled researchers for some time. Citrobacter rodentium provides a natural model for studying A/E pathogens in vivo. Type III secretion system (T3SS) effectors are involved in microvilli effacement and deregulation of ion exchangers, leading to a concomitant decrease in water absorption. Effacement of microvilli is a cooperative function of intimin and the T3SS effectors mitochondrial-associated protein (Map), translocated intimin receptor (Tir) and $\mathrm{EspF}^{139}$, although other effectors are probably also involved. EspF contributes to tight junction disruption ${ }^{29}$ and also decreases the activity of $\mathrm{Na}^{+} / \mathrm{H}^{+}$ exchanger 3 (NHE3), a major mediator of $\mathrm{Na}^{+}$absorption ${ }^{140}$. Independently, EspG- and EspG2-induced alterations of microtubules ${ }^{141}$ affect $\mathrm{Cl}^{-}-\mathrm{OH}^{-}$exchanger activity ${ }^{142}$. Furthermore, both $\mathrm{EspF}$ and $\mathrm{EspG}$ mislocalize aquaporins ${ }^{143}$, which are the channels that are responsible for rapid water transport, and the sodium-D-glucose cotransporter $(\mathrm{SGLT} 1)^{139}$ and the intestinal serotonin transporter (SERT) ${ }^{144}$ are also inhibited. SGLT1 is a major water pump that is inhibited by a combination of intimin, Map, Tir and EspF, but inhibition is independent of effacement. SERT is involved in the uptake of serotonin, a hormone that is involved in intestinal absorption and secretion of electrolytes and fluids. In addition, connexin 43 (Cx43; also known as GJA1) hemichannels were found to be upregulated on the apical surface in response to $C$. rodentium infection, resulting in increased luminal water content ${ }^{145}$. So far, no T3SS effectors have been found to be associated with the inhibition of SERT, despite this being T3SS-dependent, or with the upregulation of $\mathrm{Cx} 43$. Collectively, these disruptions in water absorption and ion balance result in $\mathrm{A} / \mathrm{E}$ pathogen-mediated diarrhoea, although the exact contribution of each component to diarrhoea is not known. and cause actin polymerization and ruffle formation for bacterial entry ${ }^{73}$. RAC1, which can promote membrane ruffle formation, may be activated by IpgB1 mimicry of RhoG in the ELMO-DOCK180 (engulfment and cell motility-dedicator of cytokinesis protein 1) pathway ${ }^{24,74}$ or directly, by IpgB1 guanine-nucleotide exchange factor activity $^{25}$. Other effectors, such as IpgD, IpaA and VirA, are involved in the destabilization of actin and microtubules to promote invasion into a phagosome, and escape from phagosomes is dependent on the effectors IpaB, IpaC, IpaD and $\mathrm{IpaH}_{7.8}$ (reviewed in REF. 72).

Once free in the epithelial cell cytoplasm, Shigella promote their survival by using effectors to further subvert host cell processes (FIG. 3). To prevent intestinal epithelial cell turnover, IpaB mediates cell cycle arrest by targeting MAD2L2, which is an inhibitor of anaphase $\mathrm{e}^{75}$, and OspE has been shown to interact with integrin-linked kinase (ILK) to prevent epithelial cell detachment ${ }^{76}$. Apoptosis is also prevented through $\operatorname{IpgD}^{77}$, which can stimulate phosphoinositide 3-kinase and activate Akt proteins, which regulate cell survival. These three mechanisms prevent cell death and sloughing, providing a replicative niche for Shigella to maintain an infection. To persist inside colonocytes, Shigella must also evade innate immune responses, for which they use at least four effectors. One of these effectors, OspG, was shown to bind ubiquitylated $\mathrm{E} 2$ proteins, which prevents degradation of inhibitor of nuclear factor- $\kappa \mathrm{B}(\mathrm{NF}-\kappa \mathrm{B})$ subunit- $\alpha(\mathrm{I} \kappa \mathrm{B} \alpha)$ and thus inhibits NF- $\kappa B$ activation ${ }^{78}$. Additionally, OspF is targeted to the nucleus, where it irreversibly dephosphorylates mitogen-activated protein kinases that are required for the transcription of genes that are regulated by NF- $\kappa \mathrm{B}^{79}$. $\mathrm{IpaH}_{9.8}$ is also targeted to the nucleus, where it interacts with a splicing factor that is involved in the expression of inflammatory cytokines ${ }^{80}$. The fourth effector, OspB, acts with OspF to reduce interleukin-8
(IL-8) levels by recruiting host factors that remodel chromatin $^{81}$. Collectively, these four effectors are involved in dampening inflammatory responses and therefore allowing persistence of the bacteria.

As Shigella do not have flagella, movement in the host cytosol and cell-to-cell dissemination require manipulation of the host machinery (FIG. 3). The outer-membrane protein VirG (also known as IcsA) localizes to a single pole and recruits and activates N-WASP and the ARP2/3 complex for actin polymerization ${ }^{82}$. The growth of the actin filaments pushes the bacteria through the cell. The ability of VirA to stimulate the destabilization of microtubules plays an important part in the efficient intracellular spread of Shigella by providing a channel that enables them to move throughout the cell ${ }^{83}$. As a host defence, autophagy targets cytosolic Shigella through autophagy protein 5 (ATG5) recognition of VirG. Interestingly, the secreted effector IcsB can bind VirG and sequester it, thereby avoiding another aspect of host defence ${ }^{84}$.

Enteroaggregative Escherichia coli. Although it is considered to be an emerging pathogen, EAEC is the second most common cause of travellers' diarrhoea after ETEC in both developed and developing countries. EAEC is also becoming commonly recognized as a cause of endemic and epidemic diarrhoea worldwide. Diarrhoea caused by EAEC is often watery, but it can be accompanied by mucus or blood. EAEC colonization can occur in the mucosa of both the small and large bowels, which can lead to mild inflammation in the colon ${ }^{38}$. Much like the details of its transmission and epidemiology, the understanding of EAEC and its pathogenesis is limited, in part owing to the paucity of suitable animal models and the heterogeneity of virulence factors.

The characteristic phenotype of EAEC is aggregative adhesion, which involves the formation of a stacked-brick pattern of HEp-2 cells and is mediated by the genes that are found on a family of virulence plasmids called pAA plasmids. These $100 \mathrm{~kb}$ plasmids encode the necessary genes for the biogenesis of the aggregative adherence fimbriae (AAFs), which are related to the Dr family of adhesins and mediate the adherence of EAEC to the intestinal mucosa (FIG. 4b). AAF- and flagellin-mediated adherence induces an IL-8 response, which leads to the transmigration of neutrophils ${ }^{85,86}$. Four variants of AAFs (AAF/I, AAF/II, AAF/III and Hda) have been identified ${ }^{87,88}$. The receptors for AAFs are unknown, but recent data show that AAF/II can bind fibronectin ${ }^{89}$. Fimbrial extension of the positively charged AAFs away from the negatively charged lipopolysaccharide is thought to occur through a secreted protein called dispersin ${ }^{90}$. Dispersin associates with lipopolysaccharide through electrostatic interactions and is speculated to mask the negative charge of the lipopolysaccharide, thus allowing the AAF to extend away from the bacterial surface instead of collapsing onto it. This promotes dispersion of EAEC across the intestinal mucosa, by counteracting excessive AAF-mediated aggregation between other EAEC cells.

Biofilms formed by EAEC are distinct from biofilms formed by non-pathogenic E. coli, in that they can form independently of common factors such as curli, flagella 
and antigen $43(\mathrm{Ag} 43)^{91}$. EAEC biofilms on the surface of enterocytes are encased in a thick mucus layer. EAEC is thought to be able to penetrate this mucus layer through the mucolytic activity of the SPATE $\underline{\mathrm{Pic}}^{92}$. A few genes, both plasmid-borne and chromosomal, encoding proteins that are involved in the formation of biofilms have been identified, including genes that encode a type VI secretion system ${ }^{93}$; the details, however, remain cursory.

EAEC causes mucosal damage by secreting cytotoxins, although not all toxins are found in all isolates. The plasmid-encoded toxin (Pet) is a SPATE that targets $\alpha$-fodrin (also called SPTAN1), disrupting the actin cytoskeleton, and induces exfoliation ${ }^{1}$. The toxin is internalized by a clathrin-based endocytosis mechanism and is subsequently trafficked through the endoplasmic reticulum to the cytosol ${ }^{94,95}$. Two other toxins, Shigella enterotoxin 1 (ShET1) and EAST1, can be found in other pathogenic $E$. coli isolates, and their role in pathogenesis is not completely understood.

Diffusely adherent Escherichia coli. DAEC is a heterogenous group that generates a diffuse adherence pattern on HeLa and HEp-2 cells. This pattern is mediated by proteins encoded by a family of related operons, which include both fimbrial (for example, Dr and F1845) and afimbrial (Afa) adhesins, collectively designated Afa-Dr adhesins (reviewed in REF. 96). DAEC isolates that express any of the Afa-Dr adhesins (which are referred to as Afa-Dr DAEC) colonize the small bowel and have been implicated in diarrhoea in children between the ages of 18 months and 5 years, as well as in recurring urinary tract infections (UTIs) in adults ${ }^{96}$.

All Afa-Dr adhesins interact with brush borderassociated complement decay-accelerating factor (DAF), which is found on the surface of intestinal and urinary epithelial cells (FIG. 4C). Binding to DAF results in the aggregation of DAF molecules underneath the adherent bacteria. It also triggers a $\mathrm{Ca}^{2+}$-dependent signalling cascade, which results in the elongation and damage of brush border microvilli through the disorganization of key components of the cytoskeleton ${ }^{96}$. Furthermore, along with flagella the interaction between Afa-Dr adhesins and DAF induces IL-8 secretion from enterocytes, which promotes transmigration of polymorphonuclear neutrophils (PMNs) across the mucosal epithelial layer. This stimulates the upregulation of DAF on the apical surface of epithelial cells, providing DAEC with more receptors for tighter adherence ${ }^{97}$. DAEC interactions with PMNs, mediated by Afa-Dr, lead to an accelerated rate of PMN apoptosis and a decreased rate of PMN-mediated phagocytosis $^{98}$.

A subclass of Afa-Dr fimbriae interact with members of the carcinoembryonic antigen-related cell adhesion molecule (CEACAM) family of receptors that are found on the surfaces of membranes, in particular in lipid rafts $^{96}$. Interactions with CEACAMs enhance the activation of CDC42, leading to CEACAM aggregation underneath the adherent bacteria and the effacement of the brush border microvilli99. These lesions disrupt several brush border enzymes that are involved in intestinal secretion and absorption, which may contribute to diarrhoea ${ }^{96}$. It was recently shown that interactions between Dr and CEACAMs cause CEACAM dimers to dissociate, so that Dr can interact with the monomeric form of the receptor ${ }^{100}$. This may serve as an interesting method to manipulate host pathways through the response that is mediated by the disruption of CEACAM dimers. Afa-Dr adhesin interactions with CEACAMs and with DAF may be involved in microtubule-dependent uptake of DAEC cells, following which the bacteria can survive in vacuoles ${ }^{101}$.

Unlike other pathogenic E. coli, the pathogenesis of DAEC seems to be predominately mediated through Afa-Dr adhesin interactions with host cells. Secreted autotransporter toxin (Sat) has been implicated in lesions of tight junctions that are found with Afa-Dr DAEC infection and in increased permeability ${ }^{102}$. No secretion systems or other virulence factors have been identified in typical Afa-Dr DAEC isolates.

Uropathogenic Escherichia coli. UPEC infections account for roughly $80 \%$ of all UTIs, causing cystitis in the bladder and acute pyelonephritis in the kidneys. UPEC has the challenge of moving from the intestinal tract to establish an infection in the urinary tract, where it uses peptides and amino acids as the primary carbon source for fitness ${ }^{103}$. The ability to ascend the urinary tract from the urethra to the bladder and kidneys reflects exceptional mechanisms for organ tropism, evading innate immunity and avoiding clearance by micturition. Several highly regulated virulence factors contribute to this complex pathogenesis, including multiple pili, secreted toxins (for example Sat and vacuolating autotransporter toxin (Vat)), multiple iron acquisition systems and a polysaccharide capsule (reviewed in REF. 104) (FIG. 5a).

Entry of UPEC into the urinary tract is followed by adhesion to the uroepithelium. This attachment is mediated by fimbrial adhesin $\mathrm{H}($ FimH $)$, which is found at the tip of the phase-variable type 1 pili. FimH binds to the glycosylated uroplakin Ia that coats terminally differentiated superficial facet cells in the bladder ${ }^{104}$. Interactions between FimH and uroplakin IIIa were recently found to lead to phosphorylation events that are required to stimulate unknown signalling pathways for invasion and apoptosis ${ }^{105}$. UPEC invasion is also mediated by FimH binding to $\alpha 3$ and $\beta 1$ integrins that are clustered with actin at the sites of invasion ${ }^{106}$, as well as by microtubule destabilization ${ }^{107}$. These interactions trigger local actin rearrangement by stimulating kinases and Rhofamily GTPases, which results in the envelopment and internalization of the attached bacteria. Once internalized, UPEC can rapidly replicate and form biofilm-like complexes termed intracellular bacterial communities (IBCs) or pods, which serve as transient, protective environments ${ }^{108}$. UPEC can leave the IBCs through a fluxing mechanism; motile UPEC leaves the epithelial cells and enters the lumen of the bladder ${ }^{109}$. Filamentous UPEC has also been observed fluxing out of an infected cell, looping and invading surrounding superficial cells in response to innate immune responses ${ }^{109,110}$. 
During infection, the resulting influx of PMNs causes tissue damage, and UPEC attachment and invasion results in apoptosis and exfoliation of bladder cells. In addition, sublytic concentrations of the poreforming haemolysin A ( $\underline{\text { HlyA) }}$ ) toxin can inhibit AKT activation and lead to host cell apoptosis and exfoliation $^{111}$. This breach of the superficial facet cells temporarily exposes the underlying transitional cells to invasion and dissemination of UPEC. Invading bacteria are trafficked in endocytic vesicles enmeshed with actin fibres, where replication is restricted ${ }^{112,113}$. Disruption of host actin permits rapid replication, which can lead to IBC formation in the cytosol or fluxing out of the cell. This quiescent state may act as a reservoir that is protected from host immunity and may therefore permit long-term persistence in the bladder. Interestingly, UPEC infection was recently shown to manipulate the differentiation of the urothelium ${ }^{114}$, and when urothelial turnover was chemically induced these quiescent reservoirs were able to reactivate and cause an acute infection of the bladder ${ }^{115}$. The regulation of urothelial turnover may have important implications in patient predisposition to UTIs and bladder cancer ${ }^{114}$.

UTIs that are left untreated can disseminate to the kidney in an ascending progression of disease. Ascension to the kidney is mediated by reciprocal regulation of type 1 pili and motility. Bacteria that express type 1 pili are less flagellated than those that do not, suggesting that when type 1 pili are 'switched off', UPEC can become more motile ${ }^{116}$. Futhermore, motility was shown to permit the ascension from the bladder to the kidney ${ }^{117}$. UPEC isolates that are associated with pyelonephritis often express the $\mathrm{P}$ fimbriae that adhere to Gala (1-4)Gal $\beta$ moieties of the globoseries glycolipids that are found on the surface of kidney epithelial cells ${ }^{1}$. Similarly to the inverse relationship between type 1 pili and motility, expression of $P$ fimbriae is associated with fewer flagella and repressed motility ${ }^{118}$. Crosstalk between $\mathrm{P}$ fimbriae, type 1 pili and other adhesion clusters prevents co-expression of multiple surface organelles ${ }^{119}$. The correlation between $\mathrm{P}$ fimbriae and virulence, however, remains inconclusive.

Neonatal meningitis Escherichia coli. NMEC, a common inhabitant of the gastrointestinal tract, is the most frequent cause of Gram-negative-associated meningitis in newborns. Fatality rates can approach $40 \%{ }^{1}$, and survivors are usually burdened with severe neurological sequelae. The pathogenesis of NMEC is complex, as the bacteria must enter the bloodstream through the intestine and ultimately cross the bloodbrain barrier into the central nervous system (FIG. 5b), which leads to meningeal inflammation and pleocytosis of the cerebrospinal fluid.

Initial colonization, after the bacteria have been

Pleocytosis

The presence of a higher number of cells than normal in the cerebrospinal fluid. blood), so survival in the blood is crucial. Protection from the host immune responses is provided by an antiphagocytic capsule, made up of a homopolymer of polysialic acid, and serum resistance, resulting from manipulation of the classical complement pathway by the bacterial outer-membrane protein A (OmpA) ${ }^{120}$. NMEC has also been shown to interact with immune cells: invasion of macrophages and monocytes prevents apoptosis ${ }^{121}$ and chemokine release ${ }^{122}$, providing a niche for replication before dissemination back into the blood. Maturation of dendritic cells is also inhibited by NMEC ${ }^{123}$. Recently, a lambdoid phage that encodes $\mathrm{O}$ acetyltransferase was discovered, which acetylates the $\mathrm{O}$ antigen to provide phase variation and diversity to the capsule $e^{124}$ and may therefore hide the bacteria from host defences.

The blood-brain barrier is a tight barrier formed by brain microvascular endothelial cells. Attachment of NMEC is mediated by FimH of the type 1 pili binding to CD48 (REF. 125) and by OmpA binding to its receptor, ECGP96 (REF. 126), on the surface of brain microvascular endothelial cells. Invasion occurs through the actions of Ibe proteins, FimH, OmpA and cytotoxic nectrotizing factor $1(\underline{\mathrm{CNF}})^{127}$. The receptors for the Ibe proteins are unknown, but the $67 \mathrm{kDa}$ laminin receptor (67LR; also known as RPSA) was shown to be the receptor for CNF1 (REF. 128). CNF1 is a toxin that deaminates Rho-family GTPases that are involved in myosin rearrangement ${ }^{129}$. It is possible that FimH- and OmpA-mediated attachment to brain microvascular endothelial cells may be required before translocation of CNF1 into the host cell can occur. OmpA interaction with its receptor and FimH-mediated increase of intracellular $\mathrm{Ca}^{2+}$ stimulates actin rearrangements ${ }^{125,130}$ that, along with the CNF1-stimulated myosin rearrangements, are involved in the invasion of NMEC. The K1 capsule - which is found in approximately $80 \%$ of NMEC isolates - also has a role in invasion by preventing lysosomal fusion and thus allowing delivery of live bacteria across the blood-brain barrier $^{131}$. Collectively, these mechanisms allow NMEC to penetrate the blood-brain barrier and gain access to the central nervous system, where they cause oedema, inflammation and neural damage.

\section{Conclusions and future perspectives}

It is clear that the biology of the different E. coli pathovars is complex. What makes each pathovar distinct is the subset of genes involved in the subversion of host responses and hijacking of host cell machinery. In many pathovars, the same host machinery or process is targeted but the mechanism and outcome is different. For example, EPEC and EHEC recruit the ARP2/3 complex for pedestal formation, whereas Shigella use the same machinery for entry into colonocytes and intracellular dissemination. Our improved understanding of the molecular mechanisms of E. coli pathogenesis has also uncovered new aspects of the host response to infecting pathogens. This is exemplified by our understanding of the innate immune responses that are triggered by intracellular Shigella and by a 
recent publication describing the ability of Shigella to control the necrosis of non-myeloid cells through two host components that were previously unrecognized as being part of the innate immune response ${ }^{132}$.

So what is next? Genome-sequencing efforts continue to identify more potential virulence factors, but our understanding of the interactions between virulence factors and host components remains incomplete. Much of our current knowledge is derived from in vitro observations, which do not necessarily reflect the biology in vivo ${ }^{23}$. In addition, host-pathogen interactions are not the only interfaces that occur in the gut. It is becoming clear that the microbiota play a crucial part in disease dynamics and in host-pathogen, host-commensal and commensal-pathogen interactions. For example, the microbiota have been shown to be displaced by A/E pathogens $s^{133}$, and signals from the commensal flora can also affect expression of virulence determinants ${ }^{134}$. It will be interesting to analyse the interplay of signals and responses between the host, commensals and pathogens and to see how this interplay affects the progression of disease. We must be diligent in defining all of these interfaces as we further dissect the pathogenesis of $E$. coli and therefore move towards the prevention of transmission and the development of effective vaccines and novel therapeutics to target this group of diverse pathogens.
Kaper, J. B., Nataro, J. P. \& Mobley, H. L. Pathogenic Escherichia coli. Nature Rev. Microbiol. 2, 123-140 (2004).

2. Bhavsar, A. P., Guttman, J. A. \& Finlay, B. B. Manipulation of host-cell pathways by bacteria pathogens. Nature 449, 827-834 (2007).

3. Shames, S. R., Auweter, S. D. \& Finlay, B. B. Co-evolution and exploitation of host cell signaling pathways by bacterial pathogens. Int. J. Biochem. Cell Biol. 41, 380-389 (2009).

4. Asadulghani, M. et al. The defective prophage pool of Escherichia coli O157: prophage-prophage interactions potentiate horizontal transfer of virulence determinants. PLoS Pathog. 5, e1000408 (2009). A look at the 18 prophages in EHEC 0157:H7 str. Sakai and their ability to circularize, replicate, package into phage particles and infect other cells.

5. Wirth, T. et al. Sex and virulence in Escherichia coli: an evolutionary perspective. Mol. Microbiol. 60

1136-1151 (2006)

An interesting model of how a commensal species obtains virulence traits and evolves into a pathogen.

6. Ogura, Y. et al. Comparative genomics reveal the mechanism of the parallel evolution of $\mathrm{O} 157$ and non0157 enterohemorrhagic Escherichia coli. Proc. Natl Acad. Sci. USA 106, 17939-17944 (2009).

7. Rasko, D. A. et al. The pangenome structure of Escherichia coli: comparative genomic analysis of E. coli commensal and pathogenic isolates. J. Bacteriol. 190, 6881-6893 (2008).

8. Touchon, M. et al. Organised genome dynamics in the Escherichia coli species results in highly diverse adaptive paths. PLoS Genet. 5, e1000344 (2009).

9. Lloyd, A. L., Rasko, D. A. \& Mobley, H. L. T. Defining genomic islands and uropathogen-specific genes in uropathogenic Escherichia coli. J. Bacteriol. 189, 3532-3546 (2007).

10. Iguchi, A. et al. Complete genome sequence and comparative genome analysis of enteropathogenic Escherichia coli O127:H6 strain E2348/69. J. Bacteriol. 191, 347-354 (2009).

11. Maurelli, A. T. Black holes, antivirulence genes, and gene inactivation in the evolution of bacterial pathogens. FEMS Microbiol. Lett. 267, 1-8 (2007).

12. McDaniel, T. K., Jarvis, K. G., Donnenberg, M. S. \& Kaper, J. B. A genetic locus of enterocyte effacement conserved among diverse enterobacterial pathogens. Proc. Natl Acad. Sci. USA 92, 1664-1668 (1995).

13. Deng, W. et al. Dissecting virulence: systematic and functional analyses of a pathogenicity island. Proc. Natl Acad. Sci. USA 101, 3597-3602 (2004).

14. Hyland, R. M. et al. The bundlin pilin protein of enteropathogenic Escherichia coli is an

$\mathrm{N}$-acetyllactosamine-specific lectin. Cell. Microbiol. 10, 177-187 (2008)

15. Saldaña, Z. et al. The Escherichia coli common pilus and the bundle-forming pilus act in concert during the formation of localized adherence by enteropathogenic E. coli. J. Bacteriol. 191, 3451-3461 (2009).

16. Swimm, A. I. \& Kalman, D. Cytosolic extract induces Tir translocation and pedestals in EPEC-infected red blood cells. PLoS Pathog. 4, e4 (2008).

17. Kenny, B. et al. Enteropathogenic E. coli (EPEC) transfers its receptor for intimate adherence into mammalian cells. Cell 91, 511-520 (1997).

18. Swimm, A. et al. Enteropathogenic Escherichia coli use redundant tyrosine kinases to form actin pedestals. Mol. Biol. Cell 15, 3520-3529 (2004).
19. Phillips, N., Hayward, R. D. \& Koronakis, V. Phosphorylation of the enteropathogenic E. coli receptor by the Src-family kinase c-Fyn triggers actin pedestal formation. Nature Cell Biol. 6, 618-625 (2004).

20. Bommarius, B. et al. Enteropathogenic Escherichia coli Tir is an SH2/3 ligand that recruits and activates tyrosine kinases required for pedestal formation. Mol. Microbiol 63, 1748-1768 (2007).

21. Gruenheid, S. et al. Enteropathogenic E. coli Tir binds Nck to initiate actin pedestal formation in host cells. Nature Cell Biol. 3, 856-859 (2001).

22. Kalman, D. et al. Enteropathogenic E. coli acts through WASP and Arp 2/3 complex to form actin pedestals. Nature Cell Biol. 1, 389-391 (1999).

23. Schüller, S. et al. Tir phosphorylation and Nck/N-WASP recruitment by enteropathogenic and enterohaemorrhagic Escherichia coli during ex vivo colonization of human intestinal mucosa is different to cell culture models. Cell. Microbiol. 9, 1352-1364 (2007).

24. Alto, N. M. et al. Identification of a bacterial type III effector family with $\mathrm{G}$ protein mimicry functions. Cell 124, 133-145 (2006)

25. Huang, Z et al. Structural insights into host GTPase isoform selection by a family of bacterial GEF mimics. Nature Struct. Mol. Biol. 16, 853-860 (2009). This work finds that Map, which was originally suggested to be a Rho GTPase mimic, may actually act as a guanine-nucleotide exchange factor to activate CDC42. This activity is also demonstrated for the Shigella effectors IpgB1 and IpgB2.

26. Ma, C. et al. Citrobacter rodentium infection causes both mitochondrial dysfunction and intestinal epithelial barrier disruption in vivo: role of mitochondrial associated protein (Map). Cell. Microbiol. 8, 1669-1686 (2006).

27. Nougayrède, J. \& Donnenberg, M. S

Enteropathogenic Escherichia coli EspF is targeted to mitochondria and is required to initiate the mitochondrial death pathway. Cell. Microbiol. 6 1097-1111 (2004).

28. Quitard, S., Dean, P., Maresca, M. \& Kenny, B. The enteropathogenic Escherichia coli EspF effector molecule inhibits $\mathrm{Pl}-3$ kinase-mediated uptake independently of mitochondrial targeting. Cell. Microbiol. 8, 972-981 (2006).

29. Guttman, J. A. et al. Attaching and effacing pathogeninduced tight junction disruption in vivo. Cell. Microbiol. 8, 634-645 (2006).

30. Alto, N. M. et al. The type III effector EspF coordinates membrane trafficking by the spatiotemporal activation of two eukaryotic signaling pathways. J. Cell Biol. 178 1265-1278 (2007).

31. lizumi, Y. et al. The enteropathogenic $E$. coli effector EspB facilitates microvillus effacing and antiphagocytosis by inhibiting myosin function. Cell Host Microbe 2, 383-392 (2007).

32. Kim, J. et al. The bacterial virulence factor NleA inhibits cellular protein secretion by disrupting mammalian COPII function. Cell Host Microbe 2 160-171 (2007).

33. Thanabalasuriar, A. et al. The bacterial virulence factor $\mathrm{NleA}$ is required for the disruption of intestinal tight junctions by enteropathogenic Escherichia coli. Cell. Microbiol. 27 Apr 2009 (doi: 10.1111/j. 1462-5822.2009.01376.x)

34. Marchès, O. et al. EspJ of enteropathogenic and enterohaemorrhagic Escherichia coli inhibits opsonophagocytosis. Cell. Microbiol. 10, 1104-1115 (2008).
35. Samba-Louaka, A. et al. Bacterial cyclomodulin Cif blocks the host cell cycle by stabilizing the cyclindependent kinase inhibitors p21 and p27. Cell. Microbiol. 10, 2496-2508 (2008).

36. Samba-Louaka, A., Nougayrède, J., Watrin, C., Oswald, E. \& Taieb, F. The enteropathogenic E. coli effector Cif induces delayed apoptosis in epithelial cells. Infect. Immun. 77, 5471-5477 (2009).

37. Dean, P. \& Kenny, B. The effector repertoire of enteropathogenic $E$. coli: ganging up on the host cell. Curr. Opin. Microbiol. 12, 101-109 (2009).

38. Nataro, J. P. \& Kaper, J. B. Diarrheagenic Escherichia coli. Clin. Microbiol. Rev. 11, 142-201 (1998). A thorough review on the pathogenesis, diagnosis and epidemiology of diarrhoeagenic $E$. coli.

39. Toshima, H. et al. Enhancement of Shiga toxin production in enterohemorrhagic Escherichia coli serotype O157:H7 by DNase colicins. Appl. Environ. Microbiol. 73, 7582-7588 (2007).

40. Schüller, S., Heuschkel, R., Torrente, F., Kaper, J. B. \& Phillips, A. D. Shiga toxin binding in normal and inflamed human intestinal mucosa. Microbes Infect. 9 35-39 (2007).

41. Pruimboom-Brees, I. M. et al. Cattle lack vascular receptors for Escherichia coli O157:H7 Shiga toxins. Proc. Natl Acad. Sci. USA 97, 10325-10329 (2000).

42. Römer, W. et al. Shiga toxin induces tubular membrane invaginations for its uptake into cells. Nature 450, 670-675 (2007).

43. Kurmanova, A. et al. Structural requirements for furininduced cleavage and activation of Shiga toxin. Biochem. Biophys. Res. Commun. 357, 144-149 (2007).

44. Malyukova, I. et al. Macropinocytosis in Shiga toxin 1 uptake by human intestinal epithelial cells and transcellular transcytosis. Am. J. Physiol. Gastrointest. Liver Physiol. 296, G78-92 (2009).

45. Schüller, S., Frankel, G. \& Phillips, A. D. Interaction of Shiga toxin from Escherichia coli with human intestinal epithelial cell lines and explants: Stx2 induces epithelial damage in organ culture. Cell. Microbiol. 6 , 289-301 (2004).

46. Gobert, A. P. et al. Shiga toxin produced by enterohemorrhagic Escherichia coli inhibits PI3K/NF-kB signaling pathway in globotriaosylceramide-3-negative human intestinal epithelial cells. J. Immunol. 178 8168-8174 (2007)

47. Low, A. S. et al. Analysis of fimbrial gene clusters and their expression in enterohaemorrhagic Escherichia coli O157:H7. Environ. Microbiol. 8, 1033-1047 (2006).

48. Xicohtencatl-Cortes, J. et al. The type 4 pili of enterohemorrhagic Escherichia coli O157:H7 are multipurpose structures with pathogenic attributes. J. Bacteriol. 191, 411-421 (2009).

49. Erdem, A. L., Avelino, F., Xicohtencatl-Cortes, J. \& Girón, J. A. Host protein binding and adhesive properties of $\mathrm{H} 6$ and $\mathrm{H} 7$ flagella of attaching and effacing Escherichia coli. J. Bacteriol. 189 7426-7435 (2007).

50. Rendón, M. A. et al. Commensal and pathogenic Escherichia coli use a common pilus adherence factor for epithelial cell colonization. Proc. Natl Acad. Sci. USA 104, 10637-10642 (2007).

51. Robinson, C. M., Sinclair, J. F., Smith, M. J. \& O'Brien, A. D. Shiga toxin of enterohemorrhagic Escherichia coli type O157:H7 promotes intestinal colonization. Proc. Natl Acad. Sci. USA 103. 9667-9672 (2006). 
Shiga toxin is shown to increase the expression of host nucleolin, thereby providing more receptors for intimin attachment.

52. Tobe, T. et al. An extensive repertoire of type III secretion effectors in Escherichia coli O157 and the role of lambdoid phages in their dissemination. Proc. Natl Acad. Sci. USA 103, 14941-14946 (2006).

53. DeVinney, R. et al. Enterohemorrhagic Escherichia coli O157:H7 produces Tir, which is translocated to the host cell membrane but is not tyrosine phosphorylated. Infect. Immun. 67, 2389-2398 (1999).

54. Campellone, K. G., Robbins, D. \& Leong, J. M. EspF is a translocated EHEC effector that interacts with Tir and N-WASP and promotes Nck-independent actin assembly. Dev. Cell 7, 217-228 (2004).

55. Garmendia, J. et al. TccP is an enterohaemorrhagic Escherichia coli O157:H7 type III effector protein that couples Tir to the actin-cytoskeleton. Cell. Microbiol. 6, 1167-1183 (2004).

56. Weiss, S. M. et al. IRSp53 links the enterohemorrhagic E. coli effectors Tir and $\mathrm{EspF}_{\mathrm{u}}$ for actin pedestal formation. Cell Host Microbe 5, 244-258 (2009).

57. Vingadassalom, D. et al. Insulin receptor tyrosine kinase substrate links the E. coli O157:H7 actin assembly effectors Tir and $\operatorname{EspF}(\mathrm{U})$ during pedestal formation. Proc. Natl Acad. Sci. USA 106, 6754-6759 (2009).

Along with reference 56 , this study identified IRTKS as the link between Tir, TccP and pedestal formation.

58. Cheng, H., Skehan, B. M., Campellone, K. G., Leong, J. M. \& Rosen, M. K. Structural mechanism of WASP activation by the enterohaemorrhagic $E$. coli effector

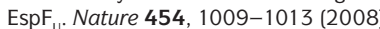

59. Sallee, N. A. et al. The pathogen protein $\operatorname{EspF}(U)$ hijacks actin polymerization using mimicry and multivalency. Nature 454, 1005-1008 (2008). Along with reference 58 , this article provides a mechanism of how TccP activates N-WASP.

60. Sal-Man, N., Biemans-Oldehinkel, E. \& Finlay, B. B. Structural microengineers: pathogenic Escherichia coli redesigns the actin cytoskeleton in host cells. Structure 17, 15-19 (2009)

61. Frankel, G. $\&$ Phillips, A. D. Attaching effacing Escherichia coli and paradigms of Tir-triggered actin polymerization: getting off the pedestal. Cell. Microbiol. 10, 549-556 (2008).

62. Hughes, D. T. \& Sperandio, V. Inter-kingdom signalling: communication between bacteria and their hosts. Nature Rev. Microbiol. 6, 111-120 (2008).

63. Turner, S. M., Scott-Tucker, A., Cooper, L. M. \& Henderson, I. R. Weapons of mass destruction virulence factors of the global killer enterotoxigenic Escherichia coli. FEMS Microbiol. Lett. 263, 10-20 (2006).

64. Jansson, L., Tobias, J., Lebens, M., Svennerholm, A. \& Teneberg, S. The major subunit, CfaB, of colonization factor antigen i from enterotoxigenic Escherichia coli is a glycosphingolipid binding protein. Infect. Immun. 74, 3488-3497 (2006)

65. Jansson, L. et al. Sulfatide recognition by colonization factor antigen CS6 from enterotoxigenic Escherichia coli. PLOS ONE. 4, e4487 (2009).

66. Roy, K. et al. Enterotoxigenic Escherichia coli EtpA mediates adhesion between flagella and host cells. Nature. 457, 594-598 (2009).

EtpA, which is transiently found at the tip of flagella, is found to mediate adhesion to host cells.

67. Dorsey, F. C., Fischer, J. F. \& Fleckenstein, J. M. Directed delivery of heat-labile enterotoxin by enterotoxigenic Escherichia coli. Cell. Microbiol. 8, 1516-1527 (2006).

68. Johnson, A. M., Kaushik, R. S., Francis, D. H. Fleckenstein, J. M. \& Hardwidge, P. R. Heat-labile enterotoxin promotes Escherichia coli adherence to intestinal epithelial cells. J. Bacteriol. 191, 178-186 (2009).

69. Kesty, N. C., Mason, K. M., Reedy, M., Miller, S. E. \& Kuehn, M. J. Enterotoxigenic Escherichia coli vesicles target toxin delivery into mammalian cells. $E M B O \mathrm{~J}$. 23, 4538-4549 (2004).

70. Chakraborty, K et al. Bacterial exotoxins downregulate cathelicidin (hCAP-18/LL-37) and human beta-defensin 1 (HBD-1) expression in the intestinal epithelial cells. Cell. Microbiol. 10, 2520-2537 (2008).

71. Ogawa, M., Handa, Y., Ashida, H., Suzuki, M. $\delta$ Sasakawa, C. The versatility of Shigella effectors. Nature Rev. Microbiol. 6, 11-16 (2008).
72. Schroeder, G. N. \& Hilbi, H. Molecular pathogenesis of Shigella spp.: controlling host cell signaling, invasion, and death by type III secretion. Clin. Microbiol. Rev. 21, 134-156 (2008).

73. Mounier, J. et al. The IpaC carboxyterminal effector domain mediates Src-dependent actin polymerization during Shigella invasion of epithelial cells. PLoS Pathog. 5, e1000271 (2009).

74. Handa, Y. et al. Shigella IpgB1 promotes bacterial entry through the ELMO-Dock180 machinery. Nature Cell Biol. 9, 121-128 (2007).

75. Iwai, H. et al. A bacterial effector targets Mad2L2, an APC inhibitor, to modulate host cell cycling. Cell 130 611-623 (2007).

76. Kim, M. et al. Bacteria hijack integrin-linked kinase to stabilize focal adhesions and block cell detachment. Nature 459, 578-582 (2009).

This investigation demonstrates that the OspE-ILK interaction stabilizes focal adhesions, preventing the detachment of infected epithelial cells.

77. Pendaries, C. et al. Ptdlns5P activates the host cell PI3-kinase/Akt pathway during Shigella flexneri infection. EMBO J. 25, 1024-1034 (2006).

78. Kim, D. W. et al. The Shigella flexneri effector OspG interferes with innate immune responses by targeting ubiquitin-conjugating enzymes. Proc. Natl Acad. Sci. USA 102, 14046-14051 (2005).

79. Arbibe, L. et al. An injected bacterial effector targets chromatin access for transcription factor NF-kB to alter transcription of host genes involved in immune responses. Nature Immunol. 8, 47-56 (2007).

80. Okuda, J. et al. Shigella effector IpaH9.8 binds to a splicing factor $\mathrm{U}_{2} \mathrm{AF}^{35}$ to modulate host immune responses. Biochem. Biophys. Res. Commun. 333 531-539 (2005).

81. Zurawski, D. V., Mumy, K. L., Faherty, C. S., McCormick, B. A. \& Maurelli, A. T. Shigella flexneri type III secretion system effectors OspB and OspF target the nucleus to downregulate the host inflammatory response via interactions with retinoblastoma protein. $\mathrm{Mol}$. Microbiol. 71, 350-368 (2009).

82. Egile, C. et al. Activation of the CDC42 effector N-WASP by the Shigella flexneri IcsA protein promotes actin nucleation by Arp $2 / 3$ complex and bacterial actinbased motility. J. Cell. Biol. 146, 1319-1332 (1999).

83. Yoshida, S. et al. Microtubule-severing activity of Shigella is pivotal for intercellular spreading. Science 314, 985-989 (2006)

84. Ogawa, M. et al. Escape of intracellular Shigella from autophagy. Science 307, 727-731 (2005). This paper shows that IcsB binds and sequesters VirG from recognition by the host cell autophagy machinery.

85. Steiner, T. S., Nataro, J. P., Poteet-Smith, C. E., Smith, J. A. \& Guerrant, R. L. Enteroaggregative Escherichia coli expresses a novel flagellin that causes IL-8 release from intestinal epithelial cells. J. Clin. Invest. 105 , 1769-1777 (2000)

86. Harrington, S. M., Strauman, M. C., Abe, C. M. $\delta$ Nataro, J. P. Aggregative adherence fimbriae contribute to the inflammatory response of epithelial cells infected with enteroaggregative Escherichia coli. Cell. Microbiol. 7, 1565-1578 (2005)

87. Harrington, S. M., Dudley, E. G. \& Nataro, J. P. Pathogenesis of enteroaggregative Escherichia coli infection. FEMS Microbiol. Lett. 254, 12-18 (2006).

88. Boisen, N., Struve, C., Scheutz, F., Krogfelt, K. A. \& Nataro, J. P. New adhesin of enteroaggregative Escherichia coli related to the Afa/Dr/AAF family. Infect. Immun. 76, 3281-3292 (2008).

89. Farfan, M. J., Inman, K. G. \& Nataro, J. P. The major pilin subunit of the AAF/II fimbriae from enteroaggregative Escherichia coli mediates binding to extracellular matrix proteins. Infect. Immun. 76 , 4378-4384 (2008)

90. Velarde, J. J. et al. Solution structure of the novel dispersin protein of enteroaggregative Escherichia coli. Mol. Microbiol. 66, 1123-1135 (2007).

91. Sheikh, J., Hicks, S., Dall'Agnol, M., Phillips, A. D. \& Nataro, J. P. Roles for Fis and YafK in biofilm formation by enteroaggregative Escherichia coli. Mol. Microbiol. 41, 983-997 (2001).

92. Gutiérrez-Jiménez, J., Arciniega, I. ¿ Navarro-García, F The serine protease motif of Pic mediates a dosedependent mucolytic activity after binding to suga constituents of the mucin substrate. Microb. Pathog. 45, 115-123 (2008).

93. Aschtgen, M., Bernard, C. S., De Bentzmann, S., Lloubès, R. \& Cascales, E. SciN is an outer membrane lipoprotein required for type $\mathrm{VI}$ secretion in enteroaggregative Escherichia coli. J. Bacteriol. 190, 7523-7531 (2008).
94. Navarro-García, F. Canizalez-Roman, A., Burlingame, K. E., Teter, K. \& Vidal, J. E. Pet, a non-AB toxin, is transported and translocated into epithelial cells by a retrograde trafficking pathway. Infect. Immun. $\mathbf{7 5}$ 2101-2109 (2007)

95. Navarro-Garcia, F., Canizalez-Roman, A., Vidal, J. E. ¿ Salazar, M. I. Intoxication of epithelial cells by plasmidencoded toxin requires clathrin-mediated endocytosis. Microbiology 153, 2828-2838 (2007).

96. Servin, A. L. Pathogenesis of Afa/Dr diffusely adhering Escherichia coli. Clin. Microbiol. Rev. 18, 264-292 (2005).

97. Bétis, F. et al. Afa/Dr diffusely adhering Escherichia coli infection in T84 cell monolayers induces increased neutrophil transepithelial migration, which in turn promotes cytokine-dependent upregulation of decayaccelerating factor (CD55), the receptor for Afa/Dr adhesins. Infect. Immun. 71, 1774-1783 (2003).

98. Brest, P. et al. Increased rate of apoptosis and diminished phagocytic ability of human neutrophils infected with Afa/Dr diffusely adhering Escherichia coli strains. Infect. Immun. 72, 5741-5749 (2004).

99. Berger, C. N., Billker, O., Meyer, T. F., Servin, A. L. \& Kansau, I. Differential recognition of members of the carcinoembryonic antigen family by Afa/Dr adhesins of diffusely adhering Escherichia coli (Afa/Dr DAEC). Mol. Microbiol. 52, 963-983 (2004).

100. Korotkova, N. et al. Binding of Dr adhesins of Escherichia coli to carcinoembryonic antigen triggers receptor dissociation. Mol. Microbiol. 67, 420-434 (2008).

101. Guignot, J., Hudault, S., Kansau, I., Chau, I. \& Servin, A. L. Human decay-accelerating factor and CEACAM receptor-mediated internalization and intracellular lifestyle of Afa/Dr diffusely adhering Escherichia coli in epithelial cells. Infect. Immun. 77, 517-531 (2009).

102. Guignot, J., Chaplais, C., Coconnier-Polter, M. \& Servin, A. L. The secreted autotransporter toxin, Sat, functions as a virulence factor in Afa/Dr diffusely adhering Escherichia coli by promoting lesions in tight junction of polarized epithelial cells. Cell. Microbiol. 9 204-221 (2007)

103. Alteri, C. J., Smith, S. N. \& Mobley, H. L. T. Fitness of Escherichia coli during urinary tract infection requires gluconeogenesis and the TCA cycle. PLoS Pathog. 5, e 1000448 (2009).

104. Wiles, T. J., Kulesus, R. R. \& Mulvey, M. A. Origins and virulence mechanisms of uropathogenic Escherichia coli. Exp. Mol. Pathol. 85, 11-19 (2008).

105. Thumbikat, P. et al. Bacteria-induced uroplakin signaling mediates bladder response to infection. PLoS Pathog. 5, e1000415 (2009).

106. Eto, D. S., Jones, T. A., Sundsbak, J. L. \& Mulvey, M. A. Integrin-mediated host cell invasion by type 1-piliated uropathogenic Escherichia coli. PLoS Pathog. 3, e100 (2007). This investigation identifies $\alpha 3$ and $\beta 1$ integrins as receptors for FimH-mediated UPEC invasion.

107. Dhakal, B. K. \& Mulvey, M. A. Uropathogenic Escherichia coli invades host cells via an HDAC6modulated microtubule-dependent pathway. J. Biol. Chem. 284, 446-454 (2009).

108. Anderson, G. G. et al. Intracellular bacterial biofilm like pods in urinary tract infections. Science 301, 105-107 (2003).

109. Justice, S. S. et al. Differentiation and developmental pathways of uropathogenic Escherichia coli in urinary tract pathogenesis. Proc. Natl Acad. Sci. USA 101, 1333-1338 (2004).

\section{This study shows that UPEC goes through four} distinct developmental stages during IBC formation.

110. Justice, S. S., Hunstad, D. A., Seed, P. C. \& Hultgren, S. J. Filamentation by Escherichia coli subverts innate defenses during urinary tract infection. Proc. Natl Acad. Sci. USA 103, 19884-19899 (2006).

111. Wiles, T. J., Dhakal, B. K., Eto, D. S. \& Mulvey, M. A. Inactivation of host Akt/protein kinase B signaling by bacterial pore-forming toxins. Mol. Biol. Cell. 19, 1427-1438 (2008)

112. Eto, D. S., Sundsbak, J. L. \& Mulvey, M. A. Actin-gated intracellular growth and resurgence of uropathogenic Escherichia coli. Cell. Microbiol. 8, 704-717 (2006).

113. Mulvey, M. A., Schilling, J. D. \& Hultgren, S. J. Establishment of a persistent Escherichia coli reservoir during the acute phase of a bladder infection. Infect. Immun. 69, 4572-4579 (2001).

114. Mysorekar, I. U., Isaacson-Schmid, M., Walker, J. N. Mills, J. C. \& Hultgren, S. J. Bone morphogenetic protein 4 signaling regulates epithelial renewal in the urinary tract in response to uropathogenic infection. Cell Host Microbe 5, 463-475 (2009). 
115. Mysorekar, I. U. \& Hultgren, S. J. Mechanisms of uropathogenic Escherichia coli persistence and eradication from the urinary tract. Proc. Natl Acad. Sci. USA 103, 14170-14175 (2006).

116. Lane, M. C., Simms, A. N. \& Mobley, H. L. T. Complex interplay between type 1 fimbrial expression and flagellum-mediated motility of uropathogenic Escherichia coli. J. Bacteriol. 189, 5523-5533 (2007).

117. Lane, M. C., Alteri, C. J., Smith, S. N. \& Mobley, H. L. T. Expression of flagella is coincident with uropathogenic Escherichia coli ascension to the upper urinary tract. Proc. Natl Acad. Sci. USA 104, 16669-16674 (2007).

118. Simms, A. N. \& Mobley, H. L. T. PapX, a P fimbria operon-encoded inhibitor of motility in uropathogenic Escherichia coli. Infect. Immun. 76, 4833-4841 (2008).

119. Lindberg, S. et al. Regulatory interactions among adhesin gene systems of uropathogenic Escherichia coli. Infect. Immun. 76, 771-780 (2008)

120. Wooster, D. G., Maruvada, R., Blom, A. M. \& Prasadarao, N. V. Logarithmic phase Escherichia coli $\mathrm{K} 1$ efficiently avoids serum killing by promoting C4bpmediated $\mathrm{C} 3 \mathrm{~b}$ and $\mathrm{C} 4 \mathrm{~b}$ degradation. Immunology 117, 482-493 (2006).

OmpA is shown to bind C4b-binding protein (C4bp), which ultimately prevents the membrane attack complex from forming.

121. Sukumaran, S. K. Selvaraj, S. K \& Prasadarao, N. V. Inhibition of apoptosis by Escherichia coli $\mathrm{K} 1$ is accompanied by increased expression of $\mathrm{Bcl}_{\mathrm{XL}}$ and blockade of mitochondrial cytochrome $c$ release in macrophages. Infect. Immun. 72, 6012-6022 (2004).

122. Selvaraj, S. K. \& Prasadarao, N. V. Escherichia coli K inhibits proinflammatory cytokine induction in monocytes by preventing NF-kB activation. J. Leukoc. Biol. 78, 544-554 (2005)

123. Mittal, R. \& Prasadarao, N. V. Outer membrane protein A expression in Escherichia coli $\mathrm{K} 1$ is required to prevent the maturation of myeloid dendritic cells and the induction of IL-10 and TGF- $\beta$. J. Immunol. 181, 2672-2682 (2008)

124. Deszo, E. L., Steenbergen, S. M., Freedberg D. I. \& Vimr, E. R. Escherichia coli K1 polysialic acid $\mathrm{O}$-acetyltransferase gene, neuO, and the mechanism of capsule form variation involving a mobile contingency locus. Proc. Natl Acad. Sci. USA 102 5564-5569 (2005).

125. Khan, N. A., Kim, Y Shin, S. \& Kim, K. S. FimH mediated Escherichia coli K1 invasion of human brain microvascular endothelial cells. Cell. Microbiol. 9 169-178 (2007)

126. Prasadarao N V Identification of Escherichia coli outer membrane protein A receptor on human brain microvascular endothelial cells. Infect. Immun. 70, 4556-4563 (2002).

127. Kim, K. S. Mechanisms of microbial traversal of the blood-brain barrier. Nature Rev. Microbiol. 6 625-634 (2008)
128. Kim, K. J., Chung J W. \& Kim, K. S. 67-kDa laminin receptor promotes internalization of cytotoxic necrotizing factor 1 -expressing Escherichia coli K1 into human brain microvascular endothelial cells. J. Biol. Chem. 280, 1360-1368 (2005).

129. Essler, M. et al. Cytotoxic necrotizing factor 1 of Escherichia coli stimulates Rho/Rho-kinase-dependent myosin light-chain phosphorylation without inactivating myosin light-chain phosphatase in endothelial cells. Infect. Immun. 71, 5188-5193 (2003).

130. Maruvada, R., Argon, Y. \& Prasadarao, N. V. Escherichia coli interaction with human brain microvascular endothelial cells induces signal transducer and activator of transcription 3 association with the C-terminal domain of Ec-gp96, the outer membrane protein A receptor for invasion. Cell. Microbiol. 10, 2326-2338 (2008).

131. Kim, K. J., Elliott, S. J., Di Cello, F., Stins, M. F. \& Kim, K. S. The K1 capsule modulates trafficking of E. colicontaining vacuoles and enhances intracellular bacterial survival in human brain microvascular endothelial cells. Cell. Microbiol. 5, 245-252 (2003).

132. Carneiro L. A M et al Shigella induces mitochondrial dysfunction and cell death in nonmyleoid cells. Cell Host Microbe 5, 123-136 (2009).

133. Lupp, C. et al. Host-mediated inflammation disrupts the intestinal microbiota and promotes the overgrowt of Enterobacteriaceae. Cell Host Microbe 2, 119-129 (2007).

134. de Sablet, T. et al. Human microbiota-secreted factors inhibit shiga toxin synthesis by enterohemorrhagic Escherichia coli O157:H7. Infect. Immun. 77, 783-790 (2009).

135. Barnich, N. et al. CEACAM6 acts as a receptor for adherent-invasive $E$. coli, supporting ileal mucosa colonization in Crohn disease. J. Clin. Invest. 117, 1566-1574 (2007)

136. Carvalho, F. A. et al.Crohn's disease adherent-invasive Escherichia coli colonize and induce strong gut inflammation in transgenic mice expressing human CEACAM. J. Exp. Med. 206, 2179-2189 (2009). This work shows that there is persistence of AIEC and inflammation in the gut of transgenic mice expressing human CEACAMs, resulting in colitis.

137. Lapaquette, P., Glasser, A., Huett, A., Xavier, R. J. ¿ Darfeuille-Michaud, A. Crohn's disease-associated adherent-invasive $E$. coli are selectively favoured by impaired autophagy to replicate intracellularly. Cell. Microbiol. 11 Sep 2009

(doi:10.1111/j.1462-5822.2009.01381.x)

138. Rolhion, N. \& Darfeuille-Michaud, A. Adherentinvasive Escherichia coli in inflammatory bowel disease. Inflamm. Bowel. Dis. 13, 1277-1283 (2007).

139. Dean, P., Maresca, M., Schüller, S., Phillips, A. D. \& Kenny, B. Potent diarrheagenic mechanism mediated by the cooperative action of three enteropathogenic Escherichia coli-injected effector proteins. Proc. Natl Acad. Sci. USA 103, 1876-1881 (2006).
140. Hodges, K Alto, N. M., Ramaswamy, K Dudeja, P. K. $\&$ Hecht, G. The enteropathogenic Escherichia coli effector protein EspF decreases sodium hydrogen exchanger 3 activity. Cell. Microbiol. 10, 1735-1745 (2008).

141. Matsuzawa, T., Kuwae, A., Yoshida, S Sasakawa, C. \& Abe, A. Enteropathogenic Escherichia coli activates the RhoA signaling pathway via the stimulation of GEF $\mathrm{H} 1$. EMBO J. 23, 3570-3582 (2004).

142. Gill, R. K. et al. Mechanism underlying inhibition of intestinal apical $\mathrm{Cl} / \mathrm{OH}$ exchange following infection with enteropathogenic E. coli. J. Clin. Invest. 117, 428-437 (2007)

143. Guttman, J. A. et al. Aquaporins contribute to diarrhoea caused by attaching and effacing bacterial pathogens. Cell. Microbiol. 9, 131-141 (2007).

144. Esmaili, A. et al. Enteropathogenic E. coli infection inhibits intestinal serotonin transporter (SERT) function and expression. Gastroenterology $10 \mathrm{Sep}$ 2009 (doi:10.1053/j.gastro.2009.09.002).

145. Guttman, J. A. et al. Gap junction hemichannels contribute to the generation of diarrhea during infectious enteric disease. Gut 14 Oct 2009 (doi: 10.1136/gut.2008.170464).

Acknowledgements

We thank the members of the Finlay laboratory for discussion and critical reading of the manuscript. We also apologize to those authors whose works were not cited owing to space constraints. Work in B.B.F's laboratory is supported by grants from the Canadian Institutes of Health Research (CIHR), the Howard Hughes Medical Institute (HHMI), the Foundation for the National Institutes of Health, the Canadian Crohn's and Colitis Foundation and Genome Canada. M.A.C. is supported by a Canadian Association of Gastroenterology/CIHR/Ferring Pharmaceuticals fellowship.

Competing interests statement

The authors declare no competing financial interests.

DATABASES

Entrez Gene: http://www.ncbi.nlm.nih.gov/gene

cadA

Entrez Genome Project: http://www.ncbi.nlm.nih.gov/ genome project

EPEC | Escherichia coli $\mid$ UPEC str. CFT073

UniProtKB: http://www. uniprot.org

Cif $\mid$ CNF1 $\mid$ EatA $\mid$ EspF $\mid$ Esp || FimH $\mid$ HlyA $\mid$ intimin $\mid$ IpaA $\mid$ IpaB $\mid$ lpaC $\mid$ lpaD $\mid$ lpgB1 $\mid$ lpgD $\mid$ NleA $\mid$ OspB $\mid$ OspF $\mid$ OspG $\mid$ Pet $\mid$ Pic |TccP|Tia|TibA|Tir|VirA|VirC

FURTHER INFORMATION

B. Brett Finlay's homepage: http://www.finlaylab.msl.ubc.ca

SUPPLEMENTARY INFORMATION

See online article: $\underline{\text { S1 }}$ (table) $\mid \underline{\text { 2 }}$ (table)

ALL LINKS ARE ACTIVE IN THE ONLINE PDF 